\title{
Non-local Operators, Non-Archimedean Parabolic-type Equations with Variable Coefficients and Markov Processes
}

\author{
by \\ L. F. Chacón-Cortes and W. A. Zúñiga-Galindo
}

\begin{abstract}
In this article, we introduce a new class of parabolic-type pseudodifferential equations with variable coefficients over the $p$-adics. We establish the existence and uniqueness of solutions for the Cauchy problem associated with these equations. The fundamental solutions of these equations are connected with Markov processes. Some of these equations are related to new models of complex systems.
\end{abstract}

2010 Mathematics Subject Classification: Primary 35K90, 60J25; Secondary 26E30. Keywords: parabolic-type equations, ultradiffusion, Markov processes, $p$-adic fields, nonArchimedean analysis.

\section{$\S 1$. Introduction}

In [3]-[4], Avetisov et al. introduced a new class of models for complex systems based on $p$-adic analysis. In these models the time-evolution of a complex system is described by a $p$-adic master equation (a parabolic-type pseudodifferential equation) which controls the time-evolution of a transition function of a Markov process on an ultrametric space, and this stochastic process is used to describe the dynamics of the system in the space of configuration states which is approximated by an ultrametric space $\left(\mathbb{Q}_{p}\right)$.

In [7], the present authors introduced a new type of non-local operators which are naturally connected with parabolic-type pseudodifferential equations. Build-

Communicated by T. Kumagi. Received May 25, 2014. Revised September 30, 2014, and October 4, 2014 .

L. F. Chacón-Cortes, W. A. Zúñiga-Galindo: Centro de Investigación y de Estudios Avanzados del Instituto Politécnico Nacional, Departamento de Matemáticas, Unidad Querétaro, Libramiento Norponiente \#2000, Fracc. Real de Juriquilla, Santiago de Querétaro, Qro. 76230, México; e-mail: fchaconc@math.cinvestav.edu.mx, wazuniga@math.cinvestav.edu.mx

Current address of L. F. Chacón-Cortes: Departamento de Matématicas, Universidad Javeriana, Carrera 7a No 43-82, Oficina 604, Bogotá, Colombia;

e-mail: leonardo.chacon@javeriana.edu.co 
ing up on [7] and [14], in this article, we introduce a new class of parabolictype pseudodifferential equations with variable coefficients, which contains the one-dimensional $p$-adic heat equation of [17], the equations studied by Kochubei [14], and the equations studied by Rodríguez-Vega [15]. Our theory is not applicable to the equations studied in [6], [18]. We establish the existence and uniqueness of solutions for the Cauchy problem for such equations (see Theorems 4.1, 5.5, 6.3). We show that the fundamental solutions of these equations are transition density functions of Markov processes (see Theorem 7.4). Finally, we study the well-posedness of the Cauchy problem (see Theorem 8.1).

On the other hand, stochastic processes on $p$-adic spaces, or more generally on ultrametric spaces, have been studied extensively (see e.g. [1], [3], [4], [7], [9], [12], [13], [14], [17], [18], and the references therein). In [1] and [13] a very large class of stochastic processes was constructed on treelike graphs, which includes the $p$-adics. This type of constructions does not need algebraic, topological or analytical properties of the $p$-adic numbers, it uses only the hierarchical structure of the $p$-adics. The construction of our stochastic processes does not rely on the hierarchical structure of the $p$-adics, but on some strong analytical properties of and methods for the $p$-adics. There are some recent developments on analysis of jump processes (that correspond to non-local operators) using Dirichlet forms on metric measure spaces, not necessarily on $p$-adic spaces (see e.g. [5], [8]). The last reference discusses heat kernel estimates for "mixtures of stable-like processes" (see Example 2.3 there) on some metric measure spaces, similar to what is discussed in Section 4 of this article.

\section{§2. Preliminaries}

In this section we fix the notation and collect some basic results of $p$-adic analysis that we will use throughout the article. For a detailed exposition the reader may consult [2], [17].

\section{$\S 2.1$. The field of $p$-adic numbers}

Along this article $p$ will denote a prime number. The field $\mathbb{Q}_{p}$ of $p$-adic numbers is defined as the completion of the field $\mathbb{Q}$ of rational numbers with respect to the $p$-adic norm $|\cdot|_{p}$, which is defined as

$$
|x|_{p}= \begin{cases}0 & \text { if } x=0, \\ p^{-\gamma} & \text { if } x=p^{\gamma} \frac{a}{b},\end{cases}
$$

where $a$ and $b$ are integers coprime with $p$. The integer $\gamma:=\operatorname{ord}(x)$, with $\operatorname{ord}(0):=$ $+\infty$, is called the $p$-adic order of $x$. We extend the $p$-adic norm to $\mathbb{Q}_{p}^{n}$ by taking

$$
\|x\|_{p}:=\max _{1 \leq i \leq n}\left|x_{i}\right|_{p} \quad \text { for } x=\left(x_{1}, \ldots, x_{n}\right) \in \mathbb{Q}_{p}^{n} .
$$


We define $\operatorname{ord}(x)=\min _{1 \leq i \leq n} \operatorname{ord}\left(x_{i}\right)$; then $\|x\|_{p}=p^{-\operatorname{ord}(x)}$. The set $\left(\mathbb{Q}_{p}^{n},\|\cdot\|_{p}\right)$ is a complete ultrametric space. As a topological space, $\mathbb{Q}_{p}$ is homeomorphic to a Cantor-like subset of the real line.

Any $p$-adic number $x \neq 0$ has a unique expansion $x=p^{\operatorname{ord}(x)} \sum_{j=0}^{\infty} x_{j} p^{j}$, where $x_{j} \in\{0,1, \ldots, p-1\}$ and $x_{0} \neq 0$. By using this expansion, we define the fractional part of $x \in \mathbb{Q}_{p}$, denoted $\{x\}_{p}$, as the rational number

$$
\{x\}_{p}= \begin{cases}0 & \text { if } x=0 \text { or ord }(x) \geq 0, \\ p^{\operatorname{ord}(x)} \sum_{j=0}^{-\operatorname{ord}(x)-1} x_{j} p^{j} & \text { if } \operatorname{ord}(x)<0 .\end{cases}
$$

For $\gamma \in \mathbb{Z}$, denote by $B_{\gamma}^{n}(a)=\left\{x \in \mathbb{Q}_{p}^{n}:\|x-a\|_{p} \leq p^{\gamma}\right\}$ the ball of radius $p^{\gamma}$ with center at $a=\left(a_{1}, \ldots, a_{n}\right) \in \mathbb{Q}_{p}^{n}$, and take $B_{\gamma}^{n}(0):=B_{\gamma}^{n}$. Notice that $B_{\gamma}^{n}(a)=B_{\gamma}\left(a_{1}\right) \times \cdots \times B_{\gamma}\left(a_{n}\right)$, where $B_{\gamma}\left(a_{i}\right):=\left\{x \in \mathbb{Q}_{p}:\left|x-a_{i}\right|_{p} \leq p^{\gamma}\right\}$ is the one-dimensional ball of radius $p^{\gamma}$ with center at $a_{i} \in \mathbb{Q}_{p}$. The ball $B_{0}^{n}$ equals the product of $n$ copies of $B_{0}:=\mathbb{Z}_{p}$, the ring of $p$-adic integers. We denote by $\Omega\left(\|x\|_{p}\right)$ the characteristic function of $B_{0}^{n}$. For more general sets, say Borel sets, we use $1_{A}(x)$ to denote the characteristic function of $A$.

\section{$\S 2.2$. The Bruhat-Schwartz space}

A complex-valued function $\varphi$ defined on $\mathbb{Q}_{p}^{n}$ is called locally constant if for any $x \in \mathbb{Q}_{p}^{n}$ there exists an integer $l=l(x) \in \mathbb{Z}$ such that

$$
\varphi\left(x+x^{\prime}\right)=\varphi(x) \quad \text { for } x^{\prime} \in B_{l}^{n} .
$$

The set of all locally constant functions $\varphi$ for which the integer $l(x)$ is independent of $x$ form a $\mathbb{C}$-vector space denoted by $\widetilde{\mathcal{E}}\left(\mathbb{Q}_{p}^{n}\right)=: \widetilde{\mathcal{E}}$. Given $\varphi \in \widetilde{\mathcal{E}}$, we call the largest possible $l=l(\varphi)$ the parameter of local constancy of $\varphi$. A function $\varphi: \mathbb{Q}_{p}^{n} \rightarrow \mathbb{C}$ is called a Bruhat-Schwartz function (or a test function) if it is locally constant with compact support. The $\mathbb{C}$-vector space of Bruhat-Schwartz functions is denoted by $S\left(\mathbb{Q}_{p}^{n}\right)=: S$. Notice that $S \subset \widetilde{\mathcal{E}}$.

Let $S^{\prime}\left(\mathbb{Q}_{p}^{n}\right):=S^{\prime}$ denote the set of all functionals (distributions) on $S\left(\mathbb{Q}_{p}^{n}\right)$. All functionals on $S\left(\mathbb{Q}_{p}^{n}\right)$ are continuous.

Set $\Psi(y)=\exp \left(2 \pi i\{y\}_{p}\right)$ for $y \in \mathbb{Q}_{p}$. The map $\Psi(\cdot)$ is an additive character on $\mathbb{Q}_{p}$, i.e. a continuous map from $\mathbb{Q}_{p}$ into the unit circle satisfying $\Psi\left(y_{0}+y_{1}\right)=$ $\Psi\left(y_{0}\right) \Psi\left(y_{1}\right)$ for all $y_{0}, y_{1} \in \mathbb{Q}_{p}$.

\section{$\S 2.3$. Fourier transform}

Given $\xi=\left(\xi_{1}, \ldots, \xi_{n}\right), x=\left(x_{1}, \ldots, x_{n}\right) \in \mathbb{Q}_{p}^{n}$, we set $\xi \cdot x:=\sum_{j=1}^{n} \xi_{j} x_{j}$. The Fourier transform of $\varphi \in S\left(\mathbb{Q}_{p}^{n}\right)$ is defined as

$$
(\mathcal{F} \varphi)(\xi)=\int_{\mathbb{Q}_{p}^{n}} \Psi(-\xi \cdot x) \varphi(x) d^{n} x \quad \text { for } \xi \in \mathbb{Q}_{p}^{n},
$$


where $d^{n} x$ is the Haar measure on $\mathbb{Q}_{p}^{n}$ normalized by the condition $\operatorname{vol}\left(B_{0}^{n}\right)=1$. The Fourier transform is a linear isomorphism from $S\left(\mathbb{Q}_{p}^{n}\right)$ onto itself satisfying $(\mathcal{F}(\mathcal{F} \varphi))(\xi)=\varphi(-\xi)$. We will also use the notation $\mathcal{F}_{x \rightarrow \xi} \varphi$ and $\widehat{\varphi}$ for the Fourier transform of $\varphi$.

The Fourier transform $\mathcal{F}[T]$ of a distribution $T \in S^{\prime}\left(\mathbb{Q}_{p}^{n}\right)$ is defined by

$$
(\mathcal{F}[T], \varphi)=(T, \mathcal{F}[\varphi]) \quad \text { for all } \varphi \in S\left(\mathbb{Q}_{p}^{n}\right) .
$$

The Fourier transform $T \mapsto \mathcal{F}[T]$ is a linear isomorphism from $S^{\prime}\left(\mathbb{Q}_{p}^{n}\right)$ onto $S^{\prime}\left(\mathbb{Q}_{p}^{n}\right)$. Furthermore, $T=\mathcal{F}[\mathcal{F}[T](-\xi)]$.

\section{§3. A class of non-local operators}

Denote by $\mathfrak{M}_{\lambda}$, with $\lambda \geq 0$, the $\mathbb{C}$-vector space of all functions $\varphi \in \widetilde{\mathcal{E}}$ satisfying $|\varphi(x)| \leq C\left(1+\|x\|_{p}^{\lambda}\right)$. If $\varphi$ depends also on a parameter $t$, we shall say that $\varphi$ belongs to $\mathfrak{M}_{\lambda}$ uniformly with respect to $t$ if its constant $C$ and its parameter of local constancy do not depend on $t$. Notice that if $0 \leq \lambda_{1} \leq \lambda_{2}$, then $\mathfrak{M}_{0} \subseteq \mathfrak{M}_{\lambda_{1}} \subseteq$ $\mathfrak{M}_{\lambda_{2}}$, and $S\left(\mathbb{Q}_{p}^{n}\right) \subseteq \mathfrak{M}_{0}$.

Take $\mathbb{R}_{+}:=\{x \in \mathbb{R}: x \geq 0\}$, and fix a function $w_{\alpha}: \mathbb{Q}_{p}^{n} \rightarrow \mathbb{R}_{+}$with the following properties:

(i) $w_{\alpha}(y)$ is a radial (i.e. $\left.w_{\alpha}(y)=w_{\alpha}\left(\|y\|_{p}\right)\right)$ and continuous function, increasing in $\|y\|_{p}$;

(ii) $w_{\alpha}(y)=0$ if and only if $y=0$;

(iii) there exist constants $C_{0}, C_{1}>0$ and $\alpha>n$ such that

$$
C_{0}\|y\|_{p}^{\alpha} \leq w_{\alpha}\left(\|y\|_{p}\right) \leq C_{1}\|y\|_{p}^{\alpha} \quad \text { for any } y \in \mathbb{Q}_{p}^{n} .
$$

Set

$$
A_{w_{\alpha}}(\xi):=\int_{\mathbb{Q}_{p}^{n}} \frac{1-\Psi(-y \cdot \xi)}{w_{\alpha}\left(\|y\|_{p}\right)} d^{n} y .
$$

In [7], we established that the function $A_{w_{\alpha}}$ is radial, positive, continuous, $A_{w_{\alpha}}(0)=0$, and $A_{w_{\alpha}}(\xi)=A_{w_{\alpha}}\left(\|\xi\|_{p}\right)=A_{w_{\alpha}}\left(p^{-\operatorname{ord}(\xi)}\right)$ is a decreasing function of ord $(\xi)$ (cf. [7, Lemma 3.2]; the condition that $w_{\alpha}\left(\|y\|_{p}\right)$ be increasing was omitted there by error, but it is necessary). In addition, we introduce the operator

$$
\left(\mathbf{W}_{\alpha} \varphi\right)(x)=\int_{\mathbb{Q}_{p}^{n}} \frac{\varphi(x-y)-\varphi(x)}{w_{\alpha}\left(\|y\|_{p}\right)} d^{n} y, \quad \varphi \in S\left(\mathbb{Q}_{p}^{n}\right) .
$$

Lemma 3.1. If $\alpha-n>\lambda$, then $\mathbf{W}_{\alpha}$ can be extended to $\mathfrak{M}_{\lambda}$ and formula (3.1) holds. Furthermore, $\mathbf{W}_{\alpha}: \mathfrak{M}_{\lambda} \rightarrow \mathfrak{M}_{\lambda}$. 
Proof. Notice that if $\varphi \in \mathfrak{M}_{\lambda}$, there exists a constant $l=l(\varphi) \in \mathbb{Z}$ such that

$$
\left(\mathbf{W}_{\alpha} \varphi\right)(x)=\int_{\|y\|_{p} \geq p^{l}} \frac{\varphi(x-y)-\varphi(x)}{w_{\alpha}\left(\|y\|_{p}\right)} d^{n} y .
$$

We now show that $\left|\left(\mathbf{W}_{\alpha} \varphi\right)(x)\right| \leq A\left(1+\|x\|_{p}^{\lambda}\right)$. Since $\varphi \in \mathfrak{M}_{\lambda}$ and $\alpha>n$,

$$
\left|\left(\mathbf{W}_{\alpha} \varphi\right)(x)\right| \leq C \int_{\|y\|_{p} \geq p^{l}} \frac{1+\|x-y\|_{p}^{\lambda}}{\|y\|_{p}^{\alpha}} d^{n} y+C^{\prime}\left(1+\|x\|_{p}^{\lambda}\right) .
$$

Hence, it is sufficient to show that the above integral is bounded by $A\left(1+\|x\|_{p}^{\lambda}\right)$ for some positive constant $A$. If $\|x\|_{p}>\|y\|_{p}$, then

$$
\int_{\|y\|_{p} \geq p^{l}} \frac{1+\|x-y\|_{p}^{\lambda}}{\|y\|_{p}^{\alpha}} d^{n} y \leq\left(1+\|x\|_{p}^{\lambda}\right) \int_{\|y\|_{p} \geq p^{l}} \frac{1}{\|y\|_{p}^{\alpha}} d^{n} y=B\left(1+\|x\|_{p}^{\lambda}\right),
$$

where $B$ is a positive constant. If $\|x\|_{p}<\|y\|_{p}$, then $\alpha-n>\lambda$ implies

$$
\int_{\|y\|_{p} \geq p^{l}} \frac{1+\|x-y\|_{p}^{\lambda}}{\|y\|_{p}^{\alpha}} d^{n} y \leq \int_{\|y\|_{p} \geq p^{l}} \frac{1+\|y\|_{p}^{\lambda}}{\|y\|_{p}^{\alpha}} d^{n} y<\infty .
$$

If $\|x\|_{p}=\|y\|_{p} \geq p^{l}$, write $x=p^{L} u, y=p^{L} v$, with $\|v\|_{p}=\|u\|_{p}=1, L \in \mathbb{Z}$; then

$$
\begin{aligned}
\int_{\|y\|_{p}=\|x\|_{p}} \frac{1+\|x-y\|_{p}^{\lambda}}{\|y\|_{p}^{\alpha}} d^{n} y & =p^{-L(n-\alpha)} \int_{\|v\|_{p}=1}\left(1+p^{-L \lambda}\|u-v\|_{p}^{\lambda}\right) d^{n} v \\
& \leq A\left(\|x\|_{p}^{-(\alpha-n)}+\|x\|_{p}^{-(\alpha-n-\lambda)}\right) \leq A^{\prime}(p, l, \alpha, n, \lambda),
\end{aligned}
$$

where $A, A^{\prime}$ are positive constants. Finally, by (3.2), $\mathbf{W}_{\alpha} \varphi$ is locally constant.

\section{$\S 4$. Parabolic-type equations with constant coefficients}

Consider the following Cauchy problem:

$$
\left\{\begin{array}{l}
\frac{\partial u}{\partial t}(x, t)-\kappa \cdot\left(\mathbf{W}_{\alpha} u\right)(x, t)=f(x, t), \quad x \in \mathbb{Q}_{p}^{n}, t \in(0, T], \\
u(x, 0)=\varphi(x)
\end{array}\right.
$$

where $\alpha>n, \kappa, T$ are positive constants, $\varphi \in \operatorname{Dom}\left(\mathbf{W}_{\alpha}\right):=\mathfrak{M}_{\lambda}$ with $\alpha-n>\lambda$, $f$ is continuous in $(x, t)$ and belongs to $\mathfrak{M}_{\lambda}$ uniformly with respect to $t$, and $u$ : $\mathbb{Q}_{p}^{n} \times[0, T] \rightarrow \mathbb{C}$ is an unknown function. We say that $u(x, t)$ is a solution of (4.1) if $u(x, t)$ is continuous in $(x, t), u(\cdot, t) \in \operatorname{Dom}\left(\mathbf{W}_{\alpha}\right)$ for $t \in[0, T], u(x, \cdot)$ is continuously differentiable on $(0, T], u(\cdot, t) \in \mathfrak{M}_{\lambda}$ uniformly in $t$, and $u$ satisfies (4.1) for all $t>0$.

The Cauchy problem (4.1) was studied in [7] using semigroup theory. In this article, we study this problem in the space $\mathfrak{M}_{\lambda}$, which is not contained in $L^{\rho}$ for any $\rho \in[1, \infty]$, and thus we cannot use semigroup theory (see e.g. [7, Theorem 6.5]). 
We define

$$
Z(x, t):=\int_{\mathbb{Q}_{p}^{n}} e^{-\kappa t A_{w_{\alpha}}\left(\|\xi\|_{p}\right)} \Psi(x \cdot \xi) d^{n} \xi,
$$

for $t>0$ and $x \in \mathbb{Q}_{p}^{n}$. Notice that $Z(x, t)=\mathcal{F}_{\xi \rightarrow x}^{-1}\left[e^{-\kappa t A_{w_{\alpha}}\left(\|\xi\|_{p}\right)}\right] \in L^{1} \cap L^{2}$ for $t>0$, since $C^{\prime}\|\xi\|_{p}^{\alpha-n} \leq A_{w_{\alpha}}\left(\|\xi\|_{p}\right) \leq C^{\prime \prime}\|\xi\|_{p}^{\alpha-n}$ (cf. [7, Lemma 3.4]). Furthermore, $Z(x, t) \geq 0$ for $t>0$ and $x \in \mathbb{Q}_{p}^{n}$ (cf. [7, Theorem 4.3(i)]). These functions are called heat kernels. When considering $Z(x, t)$ as a function of $x$ for $t$ fixed we will write $Z_{t}(x)$.

We set

$u_{1}(x, t):=\int_{\mathbb{Q}_{p}^{n}} Z(x-y, t) \varphi(y) d^{n} y, \quad u_{2}(x, t):=\int_{0}^{t} \int_{\mathbb{Q}_{p}^{n}} Z(x-y, t-\theta) f(y, \theta) d^{n} y d \theta$, for $\varphi, f \in \mathfrak{M}_{\lambda}$ with $\alpha-n>\lambda, 0 \leq t \leq T$, and $x \in \mathbb{Q}_{p}^{n}$. The main result of this section is the following:

Theorem 4.1. The function $u(x, t)=u_{1}(x, t)+u_{2}(x, t)$ is a solution of the Cauchy problem (4.1).

The proof requires several steps.

\section{§4.1. Claim: $u(x, t) \in \mathfrak{M}_{\lambda}$}

In order to prove this claim, we need some preliminary results.

Remark 4.2. The function $Z_{t}(x)$ is radial since it is the inverse Fourier transform of the radial function $e^{-\kappa t A_{w_{\alpha}}}\left(\|\xi\|_{p}\right)$. Then $Z_{t}(x)$ is locally constant in $\mathbb{Q}_{p}^{n} \backslash\{0\}$. Furthermore, if $y \in \mathbb{Q}_{p}^{n}, x \in \mathbb{Q}_{p}^{n} \backslash\{0\},\|y\|_{p}<\|x\|_{p}$ and $t>0$, then $Z_{t}(x+y)$ $=Z_{t}(x)$.

Lemma 4.3. There exist positive constants $C_{1}, C_{2}$ such that:

(i) $Z(x, t) \leq C_{1} t^{-n /(\alpha-n)}$ for $t>0$ and $x \in \mathbb{Q}_{p}^{n}$;

(ii) $Z(x, t) \leq C_{2} t\|x\|_{p}^{-\alpha}$ for $t>0$ and $x \in \mathbb{Q}_{p}^{n} \backslash\{0\}$;

(iii) $Z(x, t) \leq \max \left\{2^{\alpha} C_{1}, 2^{\alpha} C_{2}\right\} t\left(\|x\|_{p}+t^{1 /(\alpha-n)}\right)^{-\alpha}$ for $t>0$ and $x \in \mathbb{Q}_{p}^{n}$;

(iv) $\int_{\mathbb{Q}_{p}^{n}} Z(x, t) d^{n} x=1$ for $t>0$.

Proof. (i) By (4.2) and [7, Lemma 3.4],

$$
Z(x, t) \leq \int_{\mathbb{Q}_{p}^{n}} e^{-\kappa t A_{w_{\alpha}}\left(\|\xi\|_{p}\right)} d^{n} \xi \leq \int_{\mathbb{Q}_{p}^{n}} e^{-C_{0} t\|\xi\|_{p}^{\alpha-n}} d^{n} \xi .
$$

Let $m$ be an integer such that $p^{m-1} \leq t^{1 /(\alpha-n)} \leq p^{m}$. Then

$$
Z(x, t) \leq \int_{\mathbb{Q}_{p}^{n}} e^{-C_{0}\left\|p^{-(m-1)} \xi\right\|_{p}^{\alpha-n}} d^{n} \xi .
$$


By changing variables via $z=p^{-(m-1)} \xi$, we have

$$
Z(x, t) \leq p^{-(m-1) n} \int_{\mathbb{Q}_{p}^{n}} e^{-C_{0}\|z\|_{p}^{\alpha-n}} d^{n} z \leq C_{1} t^{-n /(\alpha-n)} .
$$

(ii) follows from [7, Lemma 4.1].

(iii) The result is obtained from the following two inequalities. If $\|x\|_{p} \geq$ $t^{1 /(\alpha-n)}$, then $\|x\|_{p} \geq\|x\|_{p} / 2+t^{1 /(\alpha-n)} / 2$ and $\|x\|_{p}^{-\alpha} \leq 2^{\alpha}\left(\|x\|_{p}+t^{1 /(\alpha-n)}\right)^{-\alpha}$; multiplying by $C_{2} t$ and using (ii), we obtain

$$
Z(x, t) \leq 2^{\alpha} C_{2} t\left(\|x\|_{p}+t^{1 /(\alpha-n)}\right)^{-\alpha} .
$$

If $\|x\|_{p} \leq t^{1 /(\alpha-n)}$, then $\|x\|_{p} / 2+t^{1 /(\alpha-n)} / 2 \leq t^{1 /(\alpha-n)}$ and $\left(\|x\|_{p}+t^{1 /(\alpha-n)}\right)^{-\alpha} \geq$ $2^{-\alpha} t^{-\alpha /(\alpha-n)}=2^{-\alpha} t^{-1-n /(\alpha-n)}$; multiplying by $C_{1}$ and using (i) gives

$$
Z(x, t) \leq 2^{\alpha} C_{1} t\left(\|x\|_{p}+t^{1 /(\alpha-n)}\right)^{-\alpha} .
$$

(iv) By (iii), $Z_{t}(\cdot) \in L^{1}\left(\mathbb{Q}_{p}^{n}\right)$ for $t>0$. Now, the announced identity follows by applying the Fourier inversion formula.

Proposition 4.4 ([16, Proposition 2]). If $b>0,0 \leq \lambda<\alpha$, and $x \in \mathbb{Q}_{p}^{n}$, then

$$
\int_{\mathbb{Q}_{p}^{n}}\left(b+\|x-\xi\|_{p}\right)^{-\alpha-n}\|\xi\|_{p}^{\lambda} d^{n} \xi \leq C b^{-\alpha}\left(1+\|x\|_{p}^{\lambda}\right),
$$

where the constant $C$ does not depend on $b$ or $x$.

Lemma 4.5. The functions $u_{1}, u_{2}$ belong to $\mathfrak{M}_{\lambda}$ uniformly in $t$ for $\lambda+n<\alpha$.

Proof. By Lemma 4.3(iii) and Proposition 4.4,

$$
\begin{aligned}
\left|u_{1}(x, t)\right| & \leq \int_{\mathbb{Q}_{p}^{n}} Z(x-y, t)|\varphi(y)| d^{n} y \\
& \leq C \int_{\mathbb{Q}_{p}^{n}} t\left(t^{1 /(\alpha-n)}+\|x-y\|_{p}\right)^{-\alpha}\left(1+\|y\|_{p}^{\lambda}\right) d^{n} y \leq C^{\prime}\left(1+\|x\|_{p}^{\lambda}\right) .
\end{aligned}
$$

On the other hand, since $u_{1}(x, t)=\int_{\mathbb{Q}_{p}^{n}} Z(w, t) \varphi(x-w) d^{n} w, u_{1}$ is locally constant and $l\left(u_{1}\right)=l(\varphi)$ uniformly in $t$. The proof for $u_{2}$ is similar.

Remark 4.6. Notice that $u_{1}, u_{2}, \mathbf{W}_{\gamma} u_{1}, \mathbf{W}_{\gamma} u_{2} \in \mathfrak{M}_{\lambda}$ for any $\gamma$ satisfying $\lambda+n<$ $\gamma \leq \alpha$

\section{§4.2. Claim: $u(x, t)$ satisfies the initial condition}

This claim follows from Lemma 4.5 by using the following result. 
Lemma 4.7. If $\varphi \in \mathfrak{M}_{\lambda}$ with $\alpha>\lambda+n$, then

$$
\lim _{t \rightarrow 0^{+}} \int_{\mathbb{Q}_{p}^{n}} Z(x-\xi, t) \varphi(\xi) d^{n} \xi=\varphi(x) .
$$

Proof. By Lemma 4.3(iv),

$$
\int_{\mathbb{Q}_{p}^{n}} Z(x-\xi, t) \varphi(\xi) d^{n} \xi=\int_{\mathbb{Q}_{p}^{n}} Z(x-\xi, t)[\varphi(\xi)-\varphi(x)] d^{n} \xi+\varphi(x) .
$$

Now, by Lemma 4.3(iii) and the local constancy of $\varphi$,

$$
\begin{aligned}
\int_{\mathbb{Q}_{p}^{n}} Z(x-\xi, t)[\varphi(\xi)-\varphi(x)] d^{n} \xi \\
\leq C t \int_{\|x-\xi\|_{p} \geq p^{l}}\left(t^{1 /(\alpha-n)}+\|x-\xi\|_{p}\right)^{-\alpha}|\varphi(\xi)-\varphi(x)| d^{n} \xi \\
\leq C t \int_{\|z\|_{p} \geq p^{l}}\left(t^{1 /(\alpha-n)}+\|z\|_{p}\right)^{-\alpha}|\varphi(x-z)-\varphi(x)| d^{n} z \\
\leq C t \int_{\|z\|_{p} \geq p^{l}}\|z\|_{p}^{-\alpha}\left(1+\|x-z\|^{\lambda}\right) d^{n} z+C^{\prime} t|\varphi(x)| \leq t h(x) .
\end{aligned}
$$

The desired formula is obtained by letting $t \rightarrow 0^{+}$in (4.3).

\$4.3. Claim: $u(x, t)$ is a solution of the Cauchy problem (4.1)

This claim is a consequence of Corollary 4.10 and Lemmas 4.11 and 4.12. Several preliminary results are required.

Lemma 4.8. There exist positive constants $C_{3}, C_{4}$ such that:

(i) $\frac{\partial Z(x, t)}{\partial t}=-\kappa \int_{\mathbb{Q}_{p}^{n}} A_{w_{\alpha}}\left(\|\xi\|_{p}\right) e^{-\kappa t A_{w_{\alpha}}\left(\|\xi\|_{p}\right)} \Psi(x \cdot \xi) d^{n} \xi$ for $t>0$ and $x \in \mathbb{Q}_{p}^{n}$;

(ii) $\left|\frac{\partial Z(x, t)}{\partial t}\right| \leq C_{3} t^{-\alpha /(\alpha-n)}$ for $t>0$ and $x \in \mathbb{Q}_{p}^{n}$;

(iii) $\left|\frac{\partial Z(x, t)}{\partial t}\right| \leq C_{4} t\|x\|_{p}^{n-2 \alpha}$ for $t>0$ and $x \in \mathbb{Q}_{p}^{n} \backslash\{0\}$;

(iv) $\left|\frac{\partial Z(x, t)}{\partial t}\right| \leq 2^{\alpha} C_{3}\left(\|x\|_{p}+t^{1 /(\alpha-n)}\right)^{-\alpha}$ for $t>0$ and $x \in \mathbb{Q}_{p}^{n} \backslash\{0\}$.

Proof. (i) The formula is obtained by applying the Lebesgue Dominated Convergence Theorem and the fact that $-\kappa A_{w_{\alpha}}\left(\|\xi\|_{p}\right) e^{-\kappa \tau A_{w_{\alpha}}\left(\|\xi\|_{p}\right)} \Psi(x \cdot \xi) \in L^{1}\left(\mathbb{Q}_{p}^{n}\right)$ for $\tau>0$ fixed (cf. [7, Lemma 3.4]).

(ii) By using (i) and [7, Lemma 3.4],

$$
\left|\frac{\partial Z(x, t)}{\partial t}\right| \leq \int_{\mathbb{Q}_{p}^{n}} C_{1}\|\xi\|_{p}^{\alpha-n} e^{-\kappa C_{2} t\|\xi\|_{p}^{\alpha-n}} d^{n} \xi .
$$


We now pick an integer $m$ such that $p^{m-1} \leq t^{1 /(\alpha-n)} \leq p^{m}$, and proceed as in the proof of Lemma 4.3(i) to obtain

$$
\left|\frac{\partial Z(x, t)}{\partial t}\right| \leq C_{1} p^{-(m-1) n-(m-1)(\alpha-n)} \int_{\mathbb{Q}_{p}^{n}}\|z\|_{p}^{\alpha-n} e^{-\kappa C_{2}\|z\|_{p}^{\alpha-n}} d^{n} z \leq C_{3} t^{-\alpha /(\alpha-n)} .
$$

(iii) Set $\|x\|_{p}=p^{\beta}$. Now, since $A_{w_{\alpha}}\left(\|\xi\|_{p}\right) e^{-\kappa t A_{w_{\alpha}}\left(\|\xi\|_{p}\right)} \in L^{1} \cap L^{2}$ for $t>0$, it follows that $\partial Z(x, t) / \partial t \in L^{1} \cap L^{2}$ for $t>0$, and by applying the formula for the Fourier transform of a radial function, we get

$$
\begin{aligned}
& \frac{\partial Z(x, t)}{\partial t}=\|x\|_{p}^{-n} \\
& \times\left(\left(1-p^{-n}\right) \sum_{j=0}^{\infty} A_{w_{\alpha}}\left(p^{-\beta-j}\right) e^{-\kappa t A_{w_{\alpha}}\left(p^{-\beta-j}\right)} p^{-n j}-A_{w_{\alpha}}\left(p^{-\beta+1}\right) e^{-\kappa t A_{w_{\alpha}}\left(p^{-\beta+1}\right)}\right) .
\end{aligned}
$$

Now, since $A_{w_{\alpha}}(\xi)$ is a decreasing function of $\operatorname{ord}(\xi)$,

$$
\begin{aligned}
\left|\frac{\partial Z(x, t)}{\partial t}\right| & \leq\|x\|_{p}^{-n} A_{w_{\alpha}}\left(p^{-\beta+1}\right)\left|\left(1-p^{-n}\right) \sum_{j=0}^{\infty} p^{-n j}-e^{-\kappa t A_{w_{\alpha}}\left(p^{-\beta+1}\right)}\right| \\
& \leq\|x\|_{p}^{-n} A_{w_{\alpha}}\left(p^{-\beta+1}\right)\left(1-e^{-\kappa t A_{w_{\alpha}}\left(p^{-\beta+1}\right)}\right) .
\end{aligned}
$$

By using the Mean Value Theorem and [7, Lemma 3.4], we obtain

$$
\left|\frac{\partial Z(x, t)}{\partial t}\right| \leq C_{4}\|x\|_{p}^{n-2 \alpha} t
$$

(iv) If $\|x\|_{p} \leq t^{1 /(\alpha-n)}$, then $\|x\|_{p} / 2+t^{1 /(\alpha-n)} / 2 \leq t^{1 /(\alpha-n)}$ and hence $t^{-\alpha /(\alpha-n)} \leq 2^{\alpha}\left(\|x\|_{p}+t^{1 /(\alpha-n)}\right)^{-\alpha}$; multiplying by $C_{3}$ and using (ii), we get

$$
\left|\frac{\partial Z(x, t)}{\partial t}\right| \leq 2^{\alpha} C_{3}\left(\|x\|_{p}+t^{1 /(\alpha-n)}\right)^{-\alpha} .
$$

Now, if $\|x\|_{p} \geq t^{1 /(\alpha-n)}$, then by using (iii),

$$
\left|\frac{\partial Z(x, t)}{\partial t}\right| \leq C_{3}\|x\|_{p}^{-\alpha}
$$

and since $\|x\|_{p} \geq t^{1 /(\alpha-n)}$, we find that $\|x\|_{p} \geq\|x\|_{p} / 2+t^{1 /(\alpha-n)} / 2$ and so $2^{\alpha}\left(\|x\|_{p}+t^{1 /(\alpha-n)}\right)^{-\alpha} \geq\|x\|_{p}^{-\alpha}$; multiplying by $C_{3}$ and using (4.4), we have

$$
\left|\frac{\partial Z(x, t)}{\partial t}\right| \leq 2^{\alpha} C_{3}\left(\|x\|_{p}+t^{1 /(\alpha-n)}\right)^{-\alpha}
$$


Lemma 4.9. $\left(\mathbf{W}_{\gamma} Z_{t}\right)(x)$ with $\gamma \leq \alpha$ satisfies the following conditions:

(i) $\left(\mathbf{W}_{\gamma} Z_{t}\right)(x)=-\int_{\mathbb{Q}_{p}^{n}} A_{w_{\gamma}}\left(\|\xi\|_{p}\right) e^{-\kappa t A_{w_{\alpha}}\left(\|\xi\|_{p}\right)} \Psi(x \cdot \xi) d^{n} \xi$ for $t>0$ and $x \in \mathbb{Q}_{p}^{n}$;

(ii) $\left|\left(\mathbf{W}_{\gamma} Z_{t}\right)(x)\right| \leq 2^{\gamma} C\left(\|x\|_{p}+t^{1 /(\alpha-n)}\right)^{-\gamma}$ for $t>0$ and $x \in \mathbb{Q}_{p}^{n}$ and some positive constant $C$;

(iii) $\int_{\mathbb{Q}_{p}^{n}}\left(\mathbf{W}_{\gamma} Z_{t}\right)(x) d^{n} x=0$.

Proof. (i) Define

$$
Z_{t}^{(M)}(x)=\int_{\|\eta\|_{p} \leq p^{M}} \Psi(x \cdot \eta) e^{-\kappa t A_{w_{\alpha}}\left(\|\eta\|_{p}\right)} d^{n} \eta \quad \text { for } M \in \mathbb{N} .
$$

This function is locally constant on $\mathbb{Q}_{p}^{n}$. Indeed, if $\|\xi\|_{p} \leq p^{-M}$, then $Z_{t}^{(M)}(x+\xi)=$ $Z_{t}^{(M)}(x)$. Furthermore, $Z_{t}^{(M)}(x)$ is bounded, and thus $Z_{t}^{(M)} \in \mathfrak{M}_{0} \subset \operatorname{Dom}\left(\mathbf{W}_{\gamma}\right)$. We now use formula (3.1) and Fubini's Theorem to compute

$$
\begin{aligned}
\left(\mathbf{W}_{\gamma} Z_{t}^{(M)}\right)(x) & =\int_{\mathbb{Q}_{p}^{n}} \frac{Z_{t}^{(M)}(x-\xi)-Z_{t}^{(M)}(x)}{w_{\gamma}\left(\|\xi\|_{p}\right)} d^{n} \xi \\
& =\int_{\|\xi\|_{p}>p^{-M}} \int_{\|\eta\|_{p} \leq p^{M}} e^{-\kappa t A_{w_{\alpha}}\left(\|\eta\|_{p}\right)} \Psi(x \cdot \eta) \frac{\Psi(\xi \cdot \eta)-1}{w_{\gamma}\left(\|\xi\|_{p}\right)} d^{n} \eta d^{n} \xi \\
& =\int_{\|\eta\|_{p} \leq p^{M}} e^{-\kappa t A_{w_{\alpha}}\left(\|\eta\|_{p}\right)} \Psi(x \cdot \eta) \int_{\|\xi\|_{p}>p^{-M}} \frac{\Psi(\xi \cdot \eta)-1}{w_{\gamma}\left(\|\xi\|_{p}\right)} d^{n} \xi d^{n} \eta \\
& =-\int_{\|\eta\|_{p} \leq p^{M}} e^{-\kappa t A_{w_{\alpha}}\left(\|\eta\|_{p}\right)} \Psi(x \cdot \eta) A_{w_{\gamma}}\left(\|\eta\|_{p}\right) d^{n} \eta .
\end{aligned}
$$

By using the fact that $e^{-\kappa t A_{w_{\alpha}}\left(\|\cdot\|_{p}\right)} A_{w_{\gamma}}\left(\|\cdot\|_{p}\right) \in L^{1}\left(\mathbb{Q}_{p}^{n}\right)$ for $t>0$ (cf. [7, Lemma 3.4]) and the Dominated Convergence Theorem, we obtain

$$
\lim _{M \rightarrow \infty}\left(\mathbf{W}_{\gamma} Z_{t}^{(M)}\right)(x)=-\int_{\mathbb{Q}_{p}^{n}} A_{w_{\gamma}}\left(\|\eta\|_{p}\right) e^{-\kappa t A_{w_{\alpha}}\left(\|\eta\|_{p}\right)} \Psi(x \cdot \eta) d^{n} \eta .
$$

On the other hand, by fixing $x \neq 0$ and for $t>0, Z_{t}(x-\xi)-Z_{t}(x)$ is locally constant (cf. Remark 4.2), and bounded (cf. Lemma 4.3(iii)), so $\left(\mathbf{W}_{\gamma} Z_{t}\right)(x)$ is well-defined, and since $Z_{t}^{(M)}(x)$ is radial,

$$
\left(\mathbf{W}_{\gamma} Z_{t}^{(M)}\right)(x)=\int_{\|\xi\|_{p}>\|x\|_{p}} \frac{Z_{t}^{(M)}(x-\xi)-Z_{t}^{(M)}(x)}{w_{\gamma}\left(\|\xi\|_{p}\right)} d^{n} \xi,
$$

and by the Dominated Convergence Theorem, $\lim _{M \rightarrow \infty}\left(\mathbf{W}_{\gamma} Z_{t}^{(M)}\right)(x)=$ $\left(\mathbf{W}_{\gamma} Z_{t}\right)(x)$. Therefore by $(4.6)$, we have

$$
\left(\mathbf{W}_{\gamma} Z_{t}\right)(x)=-\int_{\mathbb{Q}_{p}^{n}} A_{w_{\gamma}}\left(\|\eta\|_{p}\right) e^{-\kappa t A_{w_{\alpha}}\left(\|\eta\|_{p}\right)} \Psi(x \cdot \eta) d^{n} \eta
$$

Finally, we note the right-hand side in the above formula is continuous at $x=0$. 
(ii) By (i) and [7, Lemma 3.4],

$$
\left|\left(\mathbf{W}_{\gamma} Z_{t}\right)(x)\right| \leq C_{0} \int_{\mathbb{Q}_{p}^{n}}\|\xi\|_{p}^{\gamma-n} e^{-\kappa C_{1} t\|\xi\|_{p}^{\alpha-n}} d^{n} \xi .
$$

We now pick an integer $m$ such that $p^{m-1} \leq t^{1 /(\alpha-n)} \leq p^{m}$, and proceed as in the proof of Lemma 4.3(i) to obtain

$$
\left|\left(\mathbf{W}_{\gamma} Z_{t}\right)(x)\right| \leq C t^{-\gamma /(\alpha-n)} .
$$

Now, if $\|x\|_{p} \leq t^{1 /(\alpha-n)}$, then $\|x\|_{p} / 2+t^{1 /(\alpha-n)} / 2 \leq t^{1 /(\alpha-n)}$ and consequently $t^{-\gamma /(\alpha-n)} \leq 2^{\gamma}\left(\|x\|_{p}+t^{1 /(\alpha-n)}\right)^{-\gamma}$; multiplying by $C$ and using (4.7), we have

$$
\left|\left(\mathbf{W}_{\gamma} Z_{t}\right)(x)\right| \leq 2^{\gamma} C\left(\|x\|_{p}+t^{1 /(\alpha-n)}\right)^{-\gamma} .
$$

On the other hand, let $\|x\|_{p}=p^{\beta}$, since $A_{w_{\gamma}}\left(\|\xi\|_{p}\right) e^{-\kappa t A_{w_{\alpha}}\left(\|\xi\|_{p}\right)} \in L^{1} \cap L^{2}$ for $t>0$, we have $\mathbf{W}_{\gamma} Z_{t} \in L^{1} \cap L^{2}$ for $t>0$, by proceeding as in the proof of Lemma 4.8(iii), we obtain

$$
\left|\left(\mathbf{W}_{\gamma} Z_{t}\right)(x)\right| \leq C t\|x\|_{p}^{n-\alpha-\gamma} .
$$

Now, if $\|x\|_{p} \geq t^{1 /(\alpha-n)}$, then

$$
\left|\left(\mathbf{W}_{\gamma} Z_{t}\right)(x)\right| \leq C\|x\|_{p}^{-\gamma} .
$$

If $\|x\|_{p} \geq t^{1 /(\alpha-n)}$, then $\|x\|_{p} \geq\|x\|_{p} / 2+t^{1 /(\alpha-n)} / 2$ and $2^{\gamma}\left(\|x\|_{p}+t^{1 /(\alpha-n)}\right)^{-\gamma} \geq$ $\|x\|_{p}^{-\gamma}$; multiplying by $C$ and using (4.8), we conclude that

$$
\left|\left(\mathbf{W}_{\gamma} Z_{t}\right)(x)\right| \leq 2^{\gamma} C\left(\|x\|_{p}+t^{1 /(\alpha-n)}\right)^{-\gamma} .
$$

(iii) follows from (i) by the inversion formula for the Fourier transform.

Corollary 4.10. $\frac{\partial Z(x, t)}{\partial t}=\kappa \cdot\left(\mathbf{W}_{\alpha} Z_{t}\right)(x)$ for $t>0$ and $x \in \mathbb{Q}_{p}^{n}$.

Proof. The formula follows from Lemmas 4.8(i) and 4.9(i).

Proposition 4.11. Assume that $\varphi \in \mathfrak{M}_{\lambda}$. Then:

(i) $\frac{\partial u_{1}}{\partial t}(x, t)=\int_{\mathbb{Q}_{p}^{n}} \frac{\partial Z(x-y, t)}{\partial t} \varphi(y) d^{n} y$ for $t>0$ and $x \in \mathbb{Q}_{p}^{n} \backslash\{0\}$;

(ii) $\left(\mathbf{W}_{\gamma} u_{1}\right)(x, t)=\int_{\mathbb{Q}_{p}^{n}}\left(\mathbf{W}_{\gamma} Z_{t}\right)(x-y) \varphi(y) d^{n} y$ for $n+\lambda<\gamma \leq \alpha, t>0$ and $x \in \mathbb{Q}_{p}^{n} \backslash\{0\}$.

Proof. (i) By the Mean Value Theorem, $\frac{\partial u_{1}}{\partial t}(x, t)$ equals

$$
\lim _{h \rightarrow 0} \int_{\mathbb{Q}_{p}^{n}} \frac{Z(x-y, t+h)-Z(x-y, t)}{h} \varphi(y) d^{n} y=\lim _{h \rightarrow 0} \int_{\mathbb{Q}_{p}^{n}} \frac{\partial Z(x-y, \tau)}{\partial t} \varphi(y) d^{n} y,
$$


where $\tau$ is between $t$ and $t+h$. Now, the result follows by applying the Dominated Convergence Theorem and Lemma 4.8(iv).

(ii) By Remark 4.6, if $n+\lambda<\gamma$, then $u_{1} \in \operatorname{Dom}\left(\mathbf{W}_{\gamma}\right)$ for $t>0$. Then for any $L \in \mathbb{N}$, by Fubini's Theorem (cf. Lemma 4.3(iii)), we get

$$
\begin{aligned}
& \int_{\|y\|_{p}>p^{-L}} \frac{u_{1}(x-y, t)-u_{1}(x, t)}{w_{\gamma}\left(\|y\|_{p}\right)} d^{n} y \\
&=\int_{\mathbb{Q}_{p}^{n}} \varphi(\xi) \int_{\|y\|_{p}>p^{-L}} \frac{Z_{t}(x-\xi-y)-Z_{t}(x-\xi)}{w_{\gamma}\left(\|y\|_{p}\right)} d^{n} y d^{n} \xi .
\end{aligned}
$$

We now fix a positive integer $M$ such that $\|y\|_{p}<p^{-L}<p^{-M}<\|x-\xi\|_{p}$. By Remark 4.2,

$$
\int_{\|x-\xi\|_{p}>p^{-M}} \varphi(\xi) \int_{\|y\|_{p} \leq p^{-L}} \frac{Z_{t}(x-\xi-y)-Z_{t}(x-\xi)}{w_{\gamma}\left(\|y\|_{p}\right)} d^{n} y d^{n} \xi=0 ;
$$

then

$$
\begin{aligned}
&\left(\mathbf{W}_{\gamma} u_{1}\right)(x, t)=\lim _{L \rightarrow \infty} \int_{\|y\|_{p}>p^{-L}} \frac{u_{1}(x-y, t)-u_{1}(x, t)}{w_{\gamma}\left(\|y\|_{p}\right)} d^{n} y \\
&=\int_{\|x-\xi\|_{p}>p^{-M}} \varphi(\xi)\left(\mathbf{W}_{\gamma} Z_{t}\right)(x-\xi) d^{n} \xi \\
&+ \lim _{L \rightarrow \infty} \int_{\|x-\xi\|_{p} \leq p^{-M}} \varphi(\xi) \int_{\|y\|_{p}>p^{-L}} \frac{Z_{t}(x-\xi-y)-Z_{t}(x-\xi)}{w_{\gamma}\left(\|y\|_{p}\right)} d^{n} y d^{n} \xi \\
&=\int_{\|x-\xi\|_{p}>p^{-M}} \varphi(\xi)\left(\mathbf{W}_{\gamma} Z_{t}\right)(x-\xi) d^{n} \xi+\int_{\|x-\xi\|_{p} \leq p^{-M}} \varphi(\xi)\left(\mathbf{W}_{\gamma} Z_{t}\right)(x-\xi) d^{n} \xi,
\end{aligned}
$$

where the limit was computed by using the Lebesgue Dominated Convergence Theorem and the fact that

$$
\begin{aligned}
\int_{\|x-\xi\|_{p} \leq p^{-M}} \varphi(\xi) & \int_{\|y\|_{p}>p^{-L}} \frac{Z_{t}(x-\xi-y)-Z_{t}(x-\xi)}{w_{\gamma}\left(\|y\|_{p}\right)} d^{n} y d^{n} \xi \\
& =\int_{\|x-\xi\|_{p} \leq p^{-M}} \varphi(\xi) \int_{\|y\|_{p}>p^{-M}} \frac{Z_{t}(x-\xi-y)-Z_{t}(x-\xi)}{w_{\gamma}\left(\|y\|_{p}\right)} d^{n} y d^{n} \xi
\end{aligned}
$$

because $Z_{t}(x-\xi-y)=Z_{t}(x-\xi)$ for $\|x-\xi\|_{p}>\|y\|_{p}$ (cf. Remark 4.2). The convergence of this last integral follows from Proposition 4.4.

Set $u_{2}(x, t, \tau):=\int_{\tau}^{t} \int_{\mathbb{Q}_{n}^{n}} Z(x-y, t-\theta) f(y, \theta) d^{n} y d \theta$ for $f \in \mathfrak{M}_{\lambda}$ with $\alpha-n>\lambda$, $0 \leq \tau \leq t \leq T$, and $x \in \mathbb{Q}_{p}^{n}$. By reasoning as in the proof of Lemma 4.5, we find that $u_{2}(\cdot, t, \tau) \in \mathfrak{M}_{\lambda}$ uniformly in $t$ and $\tau$. 
Proposition 4.12. Assume that $f \in \mathfrak{M}_{\lambda}$ with $\alpha-n>\lambda$. Then:

(i) $\frac{\partial u_{2}}{\partial t}(x, t, \tau)=f(x, t)+\int_{\tau^{t}}\left(\int_{\mathbb{Q}_{p}^{n}} \frac{\partial Z(x-y, t-\theta)}{\partial t}[f(y, \theta)-f(x, \theta)] d^{n} y\right) d \theta$ for $t>0$ and $x \in \mathbb{Q}_{p}^{n}$;

(ii) $\left(\mathbf{W}_{\gamma} u_{2}\right)(x, t, \tau)=\int_{\tau^{t}} \int_{\mathbb{Q}_{p}^{n}}\left(\mathbf{W}_{\gamma} Z\right)(x-y, t-\theta) f(y, \theta) d^{n} y d \theta$ for $n+\lambda<\gamma \leq \alpha$, $t>0$ and $x \in \mathbb{Q}_{p}^{n}$.

Proof. Set

$$
u_{2, h}(x, t, \tau):=\int_{\tau}^{t-h} \int_{\mathbb{Q}_{p}^{n}} Z(x-y, t-\theta) f(y, \theta) d^{n} y d \theta, \quad 0<h<t-\tau .
$$

A standard reasoning shows that

$$
\begin{aligned}
& \frac{\partial u_{2, h}}{\partial t}(x, t, \tau) \\
& \quad=\int_{\tau}^{t-h} \int_{\mathbb{Q}_{p}^{n}} \frac{\partial Z(x-y, t-\theta)}{\partial t} f(y, \theta) d^{n} y d \theta+\int_{\mathbb{Q}_{p}^{n}} Z(x-y, h) f(y, t-h) d^{n} y d \theta .
\end{aligned}
$$

This formula can be rewritten as

$$
\begin{aligned}
\frac{\partial u_{2, h}}{\partial t}(x, t, \tau)= & \int_{\tau}^{t-h} \int_{\mathbb{Q}_{p}^{n}} \frac{\partial Z(x-y, t-\theta)}{\partial t}[f(y, \theta)-f(x, \theta)] d^{n} y d \theta \\
& +\int_{\tau}^{t-h} f(x, \theta) \int_{\mathbb{Q}_{p}^{n}} \frac{\partial Z(x-y, t-\theta)}{\partial t} d^{n} y d \theta \\
& +\int_{\mathbb{Q}_{p}^{n}} Z(x-y, h)[f(y, t-h)-f(y, t)] d^{n} y+\int_{\mathbb{Q}_{p}^{n}} Z(x-y, h) f(y, t) d^{n} y .
\end{aligned}
$$

The first integral contains no singularity at $t=\theta$ due to Lemma 4.8(iv) and the local constancy of $f$. By Lemma 4.3(iv), the second integral is zero. The third integral can be written as a sum of integrals over $\left\{y \in \mathbb{Q}_{p}^{n}:\|x-y\|_{p} \geq p^{M}\right\}$ and the complement of this set; one of these integrals is estimated on the basis of the uniform continuity of $f$, while the other contains no singularity (see Lemma 4.8(iv)). Finally, the fourth integral tends to $f(x, t)$ as $h \rightarrow 0^{+}$(cf. Lemma 4.7).

(ii) By Lemma 4.5, $\mathbf{W}_{\gamma} u_{2, h}$ is well-defined for any $\gamma$ satisfying $n+\lambda<\gamma \leq \alpha$. Then, for any $L \in \mathbb{N}$, the following integral exists:

$$
\begin{aligned}
& \int_{\|\xi\|_{p} \geq p^{-L}} \frac{u_{2, h}(x-\xi, t, \tau)-u_{2, h}(x, t, \tau)}{w_{\gamma}\left(\|\xi\|_{p}\right)} d^{n} \xi \\
= & \int_{\tau}^{t-h} \int_{\mathbb{Q}_{p}^{n}} \int_{\|\xi\|_{p} \geq p^{-L}} \frac{Z(x-\xi-y, t-\theta)-Z(x-y, t-\theta)}{w_{\gamma}\left(\|\xi\|_{p}\right)} f(y, \theta) d^{n} \xi d^{n} y d \theta .
\end{aligned}
$$


On the other hand, by Fubini's Theorem,

$$
\begin{aligned}
\int_{\|\xi\|_{p} \geq p^{-L}} \frac{Z(x-\xi-y, t-\theta)-Z(x-y, t-\theta)}{w_{\gamma}\left(\|\xi\|_{p}\right)} d^{n} \xi \\
=\int_{\mathbb{Q}_{p}^{n}} \Psi((x-y) \cdot \eta) e^{-\kappa(t-\theta) A_{w_{\gamma}}\left(\|\eta\|_{p}\right)} P_{k}(\eta) d^{n} \eta,
\end{aligned}
$$

where $P_{k}(\eta)=\int_{\|\xi\|_{p} \geq p^{-L}} \frac{\Psi(-\xi \cdot \eta)-1}{w_{\gamma}\left(\|\xi\|_{p}\right)} d^{n} \xi$. A simple calculation shows that $\left|P_{k}(\eta)\right|$ $\leq C^{\prime}\|\eta\|_{p}^{\gamma-n}$, and so

$$
\int_{\|\xi\|_{p} \geq p^{-L}} \frac{Z(x-\xi-y, t-\theta)-Z(x-y, t-\theta)}{w_{\gamma}\left(\|\xi\|_{p}\right)} d^{n} \xi \leq C,
$$

where the constant does not depend on $x, t \geq h+\tau, L$. Now, by expressing the right integral of (4.9) as

$$
\begin{aligned}
& \int_{\tau}^{t-h} \int_{\|x-\xi\|_{p}>p^{-M}} \int_{\|\xi\|_{p} \geq p^{-L}} \frac{Z(x-\xi-y, t-\theta)-Z(x-y, t-\theta)}{w_{\gamma}\left(\|\xi\|_{p}\right)} f(y, \theta) d^{n} \xi d^{n} y d \theta \\
& +\int_{\tau}^{t-h} \int_{\|x-\xi\|_{p} \leq p^{-M}} \int_{\|\xi\|_{p} \geq p^{-L}} \frac{Z(x-\xi-y, t-\theta)-Z(x-y, t-\theta)}{w_{\gamma}\left(\|\xi\|_{p}\right)} f(y, \theta) d^{n} \xi d^{n} y d \theta
\end{aligned}
$$

where $M$ is a positive integer such that $\|\xi\|_{p}<p^{-L}<p^{-M}<\|x-\xi\|_{p}$, and using the same reasoning as in the final part of the proof of Proposition 4.11(ii), we obtain

$$
\left(\mathbf{W}_{\gamma} u_{2, h}\right)(x, t)=\int_{\tau}^{t-h} \int_{\mathbb{Q}_{p}^{n}}\left(\mathbf{W}_{\gamma} Z\right)(x-\xi, t-\theta) f(y, \theta) d^{n} \xi d \theta .
$$

Now, by Lemma 4.9(ii), the fact that $f \in \mathfrak{M}_{\lambda}$, Proposition 4.4, and the Dominated Convergence Theorem, we can take the limit as $h \rightarrow 0^{+}$, which completes the proof when $\gamma<\alpha$. If $\gamma=\alpha$, formula (4.10) remains valid. By using Lemma 4.9(iii), formula (4.10) can be rewritten as

$$
\left(\mathbf{W}_{\gamma} u_{2, h}\right)(x, t)=\int_{\tau}^{t-h} \int_{\mathbb{Q}_{p}^{n}}\left(\mathbf{W}_{\gamma} Z\right)(x-\xi, t-\theta)[f(y, \theta)-f(x, \theta)] d^{n} \xi d \theta
$$

Now, by using the local constancy of $f$, we can justify the passage to the limit as $h \rightarrow 0^{+}$, which completes the proof.

Remark 4.13. By Lemmas 4.3(iv) and 4.8(i), $\int_{\mathbb{Q}_{p}^{n}} \frac{\partial Z(x-y, t-\theta)}{\partial t} d^{n} y=0$, and so

$$
\frac{\partial u_{2}}{\partial t}(x, t, \tau)=f(x, t)+\int_{\tau}^{t}\left(\int_{\mathbb{Q}_{p}^{n}} \frac{\partial Z(x-y, t-\theta)}{\partial t} f(y, \theta) d^{n} y\right) d \theta
$$

for $t>0$ and $x \in \mathbb{Q}_{p}^{n}$. 


\section{§5. Parabolic-type equations with variable coefficients}

First, we fix the notation that will be used throughout this section. We assume that $\alpha>n+1$. We fix $N+1$ positive real numbers satisfying $n<\alpha_{1}<\cdots<\alpha_{N}<\alpha$. We fix $N+2$ functions $a_{k}(x, t), k=0, \ldots, N$, and $b(x, t)$ from $\mathbb{Q}_{p}^{n} \times[0, T]$ to $\mathbb{R}$, where $T$ is a positive constant. We assume that: (i) $b(x, t)$ and $a_{k}(x, t)$, for $k=0, \ldots, N$, belong (with respect to $x$ ) to $\mathfrak{M}_{0}$ uniformly with respect to $t \in[0, T]$; (ii) $a_{0}(x, t)$ satisfies the Hölder condition in $t$ with exponent $v \in(0,1)$ uniformly in $x$. We also assume the uniform parabolicity condition $a_{0}(x, t) \geq \mu>0$ and that $\alpha_{N+1}:=n+(\alpha-n)(1-v)>\alpha_{N}$. Notice that $\alpha_{N+1}<\alpha$.

Set $\widetilde{\mathbf{W}}:=\sum_{k=1}^{N} a_{k}(x, t) \mathbf{W}_{\alpha_{k}}-b(x, t) \mathbf{I}$ with domain $\mathfrak{M}_{\lambda}$ and $0 \leq \lambda+n<\alpha_{1}$. Notice that $\widetilde{\mathbf{W}}: \mathfrak{M}_{\lambda} \rightarrow \mathfrak{M}_{\lambda}$.

In this section we construct a solution for the initial value problem

$$
\left\{\begin{array}{l}
\frac{\partial u}{\partial t}(x, t)-a_{0}(x, t)\left(\mathbf{W}_{\alpha} u\right)(x, t)-(\widetilde{\mathbf{W}} u)(x, t)=f(x, t), \\
u(x, 0)=\varphi(x)
\end{array}\right.
$$

where $x \in \mathbb{Q}_{p}^{n}, t \in(0, T], \varphi \in \mathfrak{M}_{\lambda}, f(\cdot, t) \in \mathfrak{M}_{\lambda}$ uniformly with respect to $t$ with $0 \leq \lambda<\alpha_{1}-n$, and $f(x, t)$ is continuous in $(x, t)$ (if $a_{1}(x, t)=\cdots=a_{N}(x, t) \equiv 0$ then we shall assume that $0 \leq \lambda<\alpha-n)$.

\section{$\S 5.1$. Parametrized Cauchy problem}

We first study the following Cauchy problem:

$$
\left\{\begin{array}{l}
\frac{\partial u}{\partial t}(x, t)-a_{0}(y, \theta)\left(\mathbf{W}_{\alpha} u\right)(x, t)=0, \quad x \in \mathbb{Q}_{p}^{n}, t \in(0, T], \\
u(x, 0)=\varphi(x)
\end{array}\right.
$$

where $y \in \mathbb{Q}_{p}^{n}$ and $\theta>0$ are parameters. By applying the results of Section 4 with $\kappa=a_{0}(y, \theta) \geq \mu>0$, the Cauchy problem (5.2) has a fundamental solution given by

$$
Z(x, t ; y, \theta):=\int_{\mathbb{Q}_{p}^{n}} \Psi(x \cdot \xi) e^{-a_{0}(y, \theta) t A_{w_{\alpha}}\left(\|\xi\|_{p}\right)} d^{n} \xi
$$

for $t>0$ and $x \in \mathbb{Q}_{p}^{n}$.

Remark 5.1. All statements from Lemmas 4.3, 4.8, 4.9 hold for $Z(x, t ; y, \theta)$ and the constants involved do not depend on $y$ or $\theta$. Thus, we have the estimates

$$
\begin{aligned}
Z(x, t ; y, \theta) \leq C_{1} t\left(\|x\|_{p}+t^{1 /(\alpha-n)}\right)^{-\alpha} & \text { for } t>0 \\
\left|\frac{\partial Z(x, t ; y, \theta)}{\partial t}\right| \leq C_{2}\left(\|x\|_{p}+t^{1 /(\alpha-n)}\right)^{-\alpha} & \text { for } t>0
\end{aligned}
$$




$$
\left|\left(\mathbf{W}_{\gamma} Z\right)(x, t ; y, \theta)\right| \leq C_{3}\left(\|x\|_{p}+t^{1 /(\alpha-n)}\right)^{-\gamma} \quad \text { for } t>0 \text { and } \gamma \leq \alpha,
$$

and the identities

$$
\begin{aligned}
& \int_{\mathbb{Q}_{p}^{n}} Z(x, t ; y, \theta) d^{n} x=1 \quad \text { for } t>0, \\
& \frac{\partial Z(x, t ; y, \theta)}{\partial t} \\
& \quad=-a_{0}(y, \theta) \int_{\mathbb{Q}_{p}^{n}} A_{w_{\alpha}}\left(\|\xi\|_{p}\right) e^{-a_{0}(y, \theta) t A_{w_{\alpha}}\left(\|\xi\|_{p}\right)} \Psi(x \cdot \xi) d^{n} \xi \quad \text { for } t>0, \\
& \left(\mathbf{W}_{\gamma} Z\right)(x, t ; y, \theta)=-\int_{\mathbb{Q}_{p}^{n}} A_{w_{\gamma}}\left(\|\xi\|_{p}\right) e^{-a_{0}(y, \theta) t A_{w_{\alpha}}\left(\|\xi\|_{p}\right)} \Psi(x \cdot \xi) d^{n} \xi
\end{aligned}
$$

for $t>0$ and $\gamma \leq \alpha$, and

$$
\int_{\mathbb{Q}_{p}^{n}}\left(\mathbf{W}_{\gamma} Z_{t}\right)(x, t ; y, \theta) d^{n} x=0 \quad \text { for } t>0 \text { and } \gamma \leq \alpha .
$$

Lemma 5.2. There exists a positive constant $C$ such that

$$
\left|\int_{\mathbb{Q}_{p}^{n}} \frac{\partial Z(x-y ; t, y, \theta)}{\partial t} d^{n} y\right| \leq C .
$$

Proof. See [14, proof of Lemma 4.5].

\section{$\S 5.2$. Heat potentials}

We define the parameterized heat potentials as follows:

$$
u(x, t, \tau):=\int_{\tau}^{t} \int_{\mathbb{Q}_{p}^{n}} Z(x-y, t-\theta ; y, \theta) f(y, \theta) d^{n} y d \theta,
$$

where $f \in \mathfrak{M}_{\lambda}, 0 \leq \lambda<\alpha-n, f$ is continuous in $(y, \theta)$. By using the same argument given to prove Lemma 4.5 , one proves that $u \in \mathfrak{M}_{\lambda}$ uniformly in $t$ and $\tau$.

We now calculate the derivative with respect to $t$ and the action of the operator $\mathbf{W}_{\gamma}$ on $u(x, t, \tau)$ for $n+\lambda<\gamma \leq \alpha$.

Proposition 5.3. Assume that $f \in \mathfrak{M}_{\lambda}, 0 \leq \lambda<\alpha-n$, and $f$ is continuous in $(y, \theta)$. Then

(i) $\frac{\partial u(x, t, \tau)}{\partial t}=f(x, t)+\int_{\tau}^{t} \int_{\mathbb{Q}_{p}^{n}} \frac{\partial Z(x-y, t-\theta ; y, \theta)}{\partial t} f(y, \theta) d^{n} y d \theta$;

(ii) $\left(\mathbf{W}_{\gamma} u\right)(x, t, \tau)=\int_{\tau}^{t} \int_{\mathbb{Q}_{p}^{n}}\left(\mathbf{W}_{\gamma} Z\right)(x-y, t-\theta ; y, \theta) f(y, \theta) d^{n} y d \theta, \gamma \leq \alpha$.

Proof. It is a simple variation of the proof of Proposition 4.12.

The following technical result will be used later on. 
Lemma 5.4 ([14, Lemma 4.6]). Let

$$
\begin{aligned}
J(x, \xi, t, \tau) & =\int_{\tau}^{t}(t-\theta)^{-\rho / \beta}(\theta-\tau)^{-\sigma / \beta} \\
\times & \left(\int_{\mathbb{Q}_{p}^{n}}\left[(t-\theta)^{1 / \beta}+\|x-\eta\|_{p}\right]^{-n-b_{1}}\left[(\theta-\tau)^{1 / \beta}+\|\eta-\xi\|_{p}\right]^{-n-b_{2}} d^{n} \eta\right) d \theta
\end{aligned}
$$

where $x, \xi \in \mathbb{Q}_{p}^{n}, 0 \leq \tau<t, b_{1}, b_{2}>0$ and $\rho+b_{1}, \sigma+b_{2}<\beta, \beta>1$. Then

$$
\begin{aligned}
& J(x, \xi, t, \tau) \\
& \leq C\left\{B\left(1-\frac{\rho}{\beta}, 1-\frac{\sigma+b_{2}}{\beta}\right)\left[(t-\tau)^{1 / \beta}+\|x-\xi\|_{p}\right]^{-n-b_{1}}(t-\tau)^{-\left(\rho+\sigma+b_{2}-\beta\right) / \beta}\right. \\
& \left.\quad+B\left(1-\frac{\rho+b_{1}}{\beta}, 1-\frac{\sigma}{\beta}\right)\left[(t-\tau)^{1 / \beta}+\|x-\xi\|_{p}\right]^{-n-b_{2}}(t-\tau)^{-\left(\rho+\sigma+b_{1}-\beta\right) / \beta}\right\},
\end{aligned}
$$

where $C$ is a positive constant depending only on $b_{1}, b_{2}$, and $B(\cdot, \cdot)$ denotes the Archimedean Beta function.

The proof is a simple variation of that given by Kochubei for [14, Lemma 4.6].

\section{$\S 5.3$. Construction of a solution}

Theorem 5.5. The Cauchy problem (5.1) has a solution which can be represented in the form

$$
u(x, t)=\int_{0}^{t} \int_{\mathbb{Q}_{p}^{n}} \Lambda(x, t, \xi, \tau) f(\xi, \tau) d^{n} \xi d \tau+\int_{\mathbb{Q}_{p}^{n}} \Lambda(x, t, \xi, 0) \varphi(\xi) d^{n} \xi,
$$

where the fundamental solution $\Lambda(x, t, \xi, \tau), x, \xi \in \mathbb{Q}_{p}^{n}, 0 \leq \tau<t \leq T$, has the form

$$
\Lambda(x, t, \xi, \tau)=Z(x-\xi, t-\tau ; \xi, \tau)+\mathcal{W}(x, t, \xi, \tau)
$$

with

$$
\begin{aligned}
|\mathcal{W}(x, t, \xi, \tau)| \leq & C\left\{(t-\tau)^{2-\lambda /(\alpha-n)}\left[(t-\tau)^{1 /(\alpha-n)}+\|x-\xi\|_{p}\right]^{-\alpha}\right. \\
& \left.+(t-\tau) \sum_{k=1}^{N+1}\left[(t-\tau)^{1 /(\alpha-n)}+\|x-\xi\|_{p}\right]^{-\alpha_{k}}\right\} .
\end{aligned}
$$

Furthermore $Z(x, t ; y, \theta)$ satisfies the estimates (5.3)-(5.5) and (5.10).

Proof. We apply the usual parametrix method (see e.g. [11], [14]). Our proof is essentially self-contained. We look for a fundamental solution of (5.1) having the 
form (5.12) with

$$
\mathcal{W}(x, t, \xi, \tau)=\int_{\tau}^{t} \int_{\mathbb{Q}_{p}^{n}} Z(x-\eta, t-\theta ; \eta, \theta) \Phi(\eta, \theta, \xi, \tau) d^{n} \eta d \theta,
$$

and satisfying

$$
\begin{aligned}
\frac{\partial \Lambda}{\partial t}(x, t, \xi, \tau) & -a_{0}(x, t)\left(\mathbf{W}_{\alpha} \Lambda\right)(x, t, \xi, \tau) \\
& -\sum_{k=1}^{N} a_{k}(x, t)\left(\mathbf{W}_{\alpha_{k}} \Lambda\right)(x, t, \xi, \tau)+b(x, t) \Lambda(x, t, \xi, \tau)=0
\end{aligned}
$$

for $x \neq 0, t>0$. Now by using (5.12), (5.1)-(5.8) and Proposition 5.3, we have formally

$$
\begin{aligned}
& \frac{\partial Z}{\partial t}(x-\xi, t-\tau, \xi, \tau)+\Phi(x, t, \xi, \tau)+\int_{\tau}^{t} \int_{\mathbb{Q}_{p}^{n}} \frac{\partial Z(x-\eta, t-\theta ; \eta, \theta)}{\partial t} \Phi(\eta, \theta, \xi, \tau) d^{n} \eta d \theta \\
& -a_{0}(x, t)\left\{\left(\mathbf{W}_{\alpha} Z\right)(x-\xi, t-\tau ; \xi, \tau)\right. \\
& \left.+\int_{\tau}^{t} \int_{\mathbb{Q}_{p}^{n}}\left(\mathbf{W}_{\alpha} Z\right)(x-\eta, t-\theta ; \eta, \theta) \Phi(\eta, \theta, \xi, \tau) d^{n} \eta d \theta\right\} \\
& -\sum_{k=1}^{N} a_{k}(x, t)\left\{\left(\mathbf{W}_{\alpha_{k}} Z\right)(x-\xi, t-\tau ; \xi, \tau)\right. \\
& \left.+\int_{\tau}^{t} \int_{\mathbb{Q}_{p}^{n}}\left(\mathbf{W}_{\alpha_{k}} Z\right)(x-\eta, t-\theta ; \eta, \theta) \Phi(\eta, \theta, \xi, \tau) d^{n} \eta d \theta\right\} \\
& +b(x, t)\left\{Z(x-\xi, t-\tau ; \xi, \tau)+\int_{\tau}^{t} \int_{\mathbb{Q}_{p}^{n}} Z(x-\eta, t-\theta ; \eta, \theta) \Phi(\eta, \theta, \xi, \tau) d^{n} \eta d \theta\right\}=0 .
\end{aligned}
$$

By taking

$$
\begin{aligned}
R(x, t, \xi, \tau):= & \left(a_{0}(x, t)-a_{0}(\xi, \tau)\right)\left(\mathbf{W}_{\alpha} Z\right)(x-\xi, t-\tau ; \xi, \tau) \\
& +\sum_{k=1}^{N} a_{k}(x, t)\left(\mathbf{W}_{\alpha_{k}} Z\right)(x-\xi, t-\tau ; \xi, \tau)-b(x, t) Z(x-\xi, t-\tau ; \xi, \tau)
\end{aligned}
$$

one finds that $\Phi(x, t, \xi, \tau)$ satisfies the integral equation

$$
\Phi(x, t, \xi, \tau)=R(x, t, \xi, \tau)+\int_{\tau}^{t} \int_{\mathbb{Q}_{p}^{n}} R(x, t, \eta, \theta) \Phi(\eta, \theta, \xi, \tau) d^{n} \eta d \theta
$$


Now, by using (5.5) and (5.3), we obtain

$$
\begin{aligned}
|R(x, t, \xi, \tau)| \leq & C_{0}\left(\left|a_{0}(x, t)-a_{0}(\xi, \tau)\right|\left[(t-\tau)^{1 /(\alpha-n)}+\|x-\xi\|_{p}\right]^{-\alpha}\right. \\
& +\sum_{k=1}^{N}\left[(t-\tau)^{1 /(\alpha-n)}+\|x-\xi\|_{p}\right]^{-\alpha_{k}} \\
& \left.+\left[(t-\tau)^{1 /(\alpha-n)}+\|x-\xi\|_{p}\right]^{-\alpha}(t-\tau)\right) .
\end{aligned}
$$

\section{Claim A.}

$\left|a_{0}(x, t)-a_{0}(\xi, \tau)\right|\left[(t-\tau)^{1 /(\alpha-n)}+\|x-\xi\|_{p}\right]^{-\alpha} \leq C_{1}^{\prime}\left[(t-\tau)^{1 /(\alpha-n)}+\|x-\xi\|_{p}\right]^{-\alpha_{N+1}}$, where $\alpha_{N+1}=n+(\alpha-n)(1-v)>\alpha_{N}$.

The proof is based on the Hölder condition for $a_{0}(x, t)$ :

$$
\left|a_{0}(x, t)-a_{0}(\xi, \tau)\right| \leq C_{1}(t-\tau)^{v}+\left|a_{0}(x, \tau)-a_{0}(\xi, \tau)\right| .
$$

Let $l\left(a_{0}\right)$ be the parameter of local constancy of $a_{0}$. Thus, if $\|x-\xi\|_{p} \leq p^{l\left(a_{0}\right)}$, then $\left|a_{0}(x, t)-a_{0}(\xi, \tau)\right| \leq C_{1}(t-\tau)^{v}$. In the case $\|x-\xi\|_{p} \leq p^{l\left(a_{0}\right)}$, the inequality of the claim follows from the fact that $(t-\tau)^{v}\left[(t-\tau)^{1 /(\alpha-n)}+\|x-\xi\|_{p}\right]^{-\alpha+\alpha_{N+1}}$ is bounded, which in turn follows from $\lim _{t \rightarrow \tau}(t-\tau)^{v+\frac{-\alpha+\alpha}{\alpha+1}} \frac{\alpha-n}{2}=1$. In the case $\|x-\xi\|_{p}>p^{l\left(a_{0}\right)}$, by using $\left|a_{0}(x, t)-a_{0}(\xi, \tau)\right| \leq C_{0}$, the inequality follows from

$$
\left[(t-\tau)^{1 /(\alpha-n)}+\|x-\xi\|_{p}\right]^{-\alpha+\alpha_{N+1}} \leq\|x-\xi\|_{p}^{-\alpha+\alpha_{N+1}} \leq p^{\left(-\alpha+\alpha_{N+1}\right) l\left(a_{0}\right)} .
$$

\section{Claim B.}

$$
(t-\tau)\left[(t-\tau)^{1 /(\alpha-n)}+\|x-\xi\|_{p}\right]^{-\alpha} \leq C_{2}\left[(t-\tau)^{1 /(\alpha-n)}+\|x-\xi\|_{p}\right]^{-\alpha_{N+1}} .
$$

This is a consequence of the fact that $\lim _{t \rightarrow \tau}(t-\tau)^{1+\frac{-\alpha+\alpha}{\alpha+1}}=0$.

Now from (5.16), and Claims A-B, we have

$$
|R(x, t, \xi, \tau)| \leq C \sum_{k=1}^{N+1}\left[(t-\tau)^{1 /(\alpha-n)}+\|x-\xi\|_{p}\right]^{-\alpha_{k}} .
$$

We solve the integral equation (5.15) by the method of successive approximations:

$$
\Phi(x, t, \xi, \tau)=\sum_{m=1}^{\infty} R_{m}(x, t, \eta, \theta)
$$

where $R_{1} \equiv R$ and

$$
R_{m+1}(x, t, \xi, \tau)=\int_{\tau}^{t} \int_{\mathbb{Q}_{p}^{n}} R(x, t, \eta, \theta) R_{m}(\eta, \theta, \xi, \tau) d^{n} \eta d \theta \quad \text { for } m \geq 1 .
$$




\section{Claim C.}

$$
\begin{aligned}
\left|R_{m+1}(x, t, \xi, \tau)\right| \leq & C(2 N+2)^{m}(t-\tau)^{m v} \frac{(\Gamma(v))^{m+1}}{\Gamma((m+1) v)} \\
& \times \sum_{j=1}^{N+1}\left[(t-\tau)^{1 /(\alpha-n)}+\|x-\xi\|_{p}\right]^{-\alpha_{j}},
\end{aligned}
$$

for $m \geq 0$, where $\Gamma(\cdot)$ denotes the Archimedean Gamma function.

The proof of this assertion will be given later.

It follows from Claim A, by the Stirling formula, that series (5.18) is convergent and that

$$
|\Phi(x, t, \xi, \tau)| \leq C_{0} \sum_{k=1}^{N+1}\left[(t-\tau)^{1 /(\alpha-n)}+\|x-\xi\|_{p}\right]^{-\alpha_{k}} .
$$

Now (5.13) follows from (5.19) and Lemma 5.4.

Denote by $u_{1}(x, t)$ and $u_{2}(x, t)$ the first and second terms on the right-hand side of (5.11). Substituting (5.12) into (5.11), we find that

$$
\begin{aligned}
u_{1}(x, t)= & \int_{0}^{t} \int_{\mathbb{Q}_{p}^{n}} Z(x-\xi, t-\tau ; \xi, \tau) f(\xi, \tau) d^{n} \xi d \tau \\
& +\int_{0}^{t} \int_{\mathbb{Q}_{p}^{n}} Z(x-\eta, t-\theta ; \eta, \theta) F(\eta, \theta) d^{n} \eta d \theta \\
u_{2}(x, t)= & \int_{\mathbb{Q}_{p}^{n}} Z(x-\xi, t ; \xi, 0) \varphi(\xi) d^{n} \xi+\int_{0}^{t} \int_{\mathbb{Q}_{p}^{n}} Z(x-\eta, t-\theta ; \eta, \theta) G(\eta, \theta) d^{n} \eta d \theta
\end{aligned}
$$

where

$$
\begin{aligned}
& F(\eta, \theta)=\int_{0}^{\theta} \int_{\mathbb{Q}_{p}^{n}} \Phi(\eta, \theta, \xi, \tau) f(\xi, \tau) d^{n} \xi d \tau, \\
& G(\eta, \theta)=\int_{\mathbb{Q}_{p}^{n}} \Phi(\eta, \theta, \xi, 0) \varphi(\xi) d^{n} \xi .
\end{aligned}
$$

Now, by Proposition 4.4 and (5.19), it follows that

$$
|F(\eta, \theta)| \leq C_{0}\left(1+\|\eta\|_{p}^{\lambda}\right), \quad|G(\eta, \theta)| \leq C_{1}\left(1+\|\eta\|_{p}^{\lambda}\right),
$$

for all $\eta \in \mathbb{Q}_{p}^{n}$ and $\theta \in(0, T]$.

Claim D. The functions $F$ and $G$ belong to $\widetilde{\mathcal{E}}$, and their parameters of local constancy do not depend on $\theta$.

We first note that by $(5.20)-(5.21)$, it is sufficient to show that $\Phi(\cdot, \theta, \star, \tau)$ is a locally constant function on $\left(\mathbb{Q}_{p}^{\times}\right)^{n} \times \mathbb{Q}_{p}^{n}$ and that its parameter of local constancy 
does not depend on $\theta$ or $\tau$. Now, by the recursive definition of the function $\Phi$ we see that if $L$ is the parameter of local constancy for all the functions $a_{k}(\cdot, t), b(\cdot, t)$, $\left(\mathbf{W}_{\alpha_{k}} Z\right)(\cdot, t-\tau ; \star, \tau)$ and $Z(\cdot, t-\tau ; \star, \tau)$ on $\left(\mathbb{Q}_{p}^{\times}\right)^{n} \times \mathbb{Q}_{p}^{n}$, and if $\|\delta\|_{p} \leq p^{-L}$, we have $R(x+\delta, t, \xi+\delta, \tau)=R(x, t, \xi, \tau)$. Furthermore, we successively obtain

$$
\begin{aligned}
R_{m+1}(x+\delta, t, \xi+\delta, \tau) & =\int_{\tau}^{t} \int_{\mathbb{Q}_{p}^{n}} R(x+\delta, t, \eta, \theta) R_{m}(\eta, \theta, \xi+\delta, \tau) d^{n} \eta d \theta \\
& =\int_{\tau}^{t} \int_{\mathbb{Q}_{p}^{n}} R(x+\delta, t, \zeta+\delta, \theta) R_{m}(\zeta+\delta, \theta, \xi+\delta, \tau) d^{n} \zeta d \theta \\
& =R_{m+1}(x, t, \xi, \tau),
\end{aligned}
$$

so that $\Phi(x+\delta, t, \xi+\delta, \tau)=\Phi(x, t, \xi, \tau)$, and hence

$$
\begin{aligned}
F(\eta+\delta, \theta) & =\int_{0}^{\theta} \int_{\mathbb{Q}_{p}^{n}} \Phi(\eta+\delta, \theta, \xi, \tau) f(\xi, \tau) d^{n} \xi d \tau \\
& =\int_{0}^{\theta} \int_{\mathbb{Q}_{p}^{n}} \Phi(\eta+\delta, \theta, \xi+\delta, \tau) f(\xi+\delta, \tau) d^{n} \xi d \tau=F(\eta, \theta) .
\end{aligned}
$$

Similarly, $G(\eta+\delta, \theta)=G(\eta, \theta)$ when $|\delta|_{p} \leq p^{-L}$. Thus $u_{1}(\cdot, t), u_{2}(\cdot, t) \in \mathfrak{M}_{\lambda}$ uniformly in $t$. Thus the potentials in the expressions for $u_{1}(x, t), u_{2}(x, t)$ satisfy the conditions to use the differentiation formulas given in Proposition 5.3. By using these formulas along with Proposition 5.3, (5.1)-(5.8) and (5.15), one verifies after simple transformations that $u(x, t)$ is a solution of the Cauchy problem (5.1).

Let us show that $u(x, t) \rightarrow \varphi(x)$ as $t \rightarrow 0^{+}$. Due to (5.12) and (5.13), it is sufficient to verify that

$$
v(x, t):=\int_{\mathbb{Q}_{p}^{n}} Z(x-\xi, t ; \xi, 0) \varphi(\xi) d^{n} \xi \rightarrow \varphi(x) \quad \text { as } t \rightarrow 0^{+} .
$$

By (5.6), we have

$$
\begin{aligned}
v(x, t)= & \int_{\mathbb{Q}_{p}^{n}}[Z(x-\xi, t ; \xi, 0)-Z(x-\xi, t ; x, 0)] \varphi(\xi) d^{n} \xi \\
& +\int_{\mathbb{Q}_{p}^{n}} Z(x-\xi, t ; x, 0)[\varphi(\xi)-\varphi(x)] d^{n} \xi+\varphi(x) .
\end{aligned}
$$

Now, since $Z(x-\xi, t ; \cdot, 0)$ and $\varphi(\cdot)$ are locally constant functions, it follows that both integrals are actually over the set

$$
\left\{\xi \in \mathbb{Q}_{p}^{n}:\|\xi-x\|_{p} \geq p^{-L}\right\} .
$$

By applying (5.3) on this set, we see that both integrals tend to zero as $t \rightarrow 0^{+}$. 
Proof of Claim $C$. We use induction on $m$. The case $m=0$ is (5.17). We assume the case $m$ holds. Then

$$
\begin{aligned}
\left|R_{m+1}(x, t, \xi, \tau)\right| \leq \int_{\tau}^{t} \int_{\mathbb{Q}_{p}^{n}}|R(x, t, \eta, \theta)|\left|R_{m}(\eta, \theta, \xi, \tau)\right| d^{n} \eta d \theta \\
\leq C_{0}(2 N+2)^{m-1} \frac{(\Gamma(v))^{m}}{\Gamma(m v)} \sum_{j, k=1}^{N+1} \int_{\tau}^{t}(\theta-\tau)^{(m-1) v} \\
\quad \times \int_{\mathbb{Q}_{p}^{n}}\left[(\theta-\tau)^{1 /(\alpha-n)}+\|\eta-\xi\|_{p}\right]^{-\alpha_{j}}\left[(t-\theta)^{1 /(\alpha-n)}+\|x-\eta\|_{p}\right]^{-\alpha_{k}} d^{n} \eta d \theta
\end{aligned}
$$

Now by Lemma 5.4 with $-\sigma=(m-1)(\alpha-n) v, \rho=0,-n-b_{2}=-\alpha_{j},-n-b_{1}=$ $-\alpha_{k}, \beta=\alpha-n$ (notice that the condition $\alpha>n+1$ implies $\beta>1$ ), we have

$$
\begin{aligned}
& \left|R_{m+1}(x, t, \xi, \tau)\right| \\
& \leq C_{0}\left\{(2 N+2)^{m-1} \frac{(\Gamma(v))^{m}}{\Gamma(m v)} \sum_{j, k=1}^{N+1} B\left(1, \frac{\alpha+(m-1)(\alpha-n) v-\alpha_{j}}{\alpha-n}\right)\right. \\
& +B\left(\frac{\alpha-\alpha_{k}}{\alpha-n}, \frac{\alpha-n+(m-1)(\alpha-n) v}{\alpha-n}\right)\left[(t-\tau)^{1 /(\alpha-n)}+\|x-\xi\|_{p}\right]^{-\alpha_{j}} \\
& \times\left[(t-\tau)^{1 /(\alpha-n)}+\|x-\xi\|_{p}\right]^{-\alpha_{k}}(t-\tau)^{\frac{\left.(m-1)(\alpha-n) v-\alpha_{j}+\alpha\right)}{\alpha-n}} \\
& \left.\times(t-\tau)^{\frac{\left.(m-1)(\alpha-n) v-\alpha_{k}+\alpha\right)}{\alpha-n}}\right\},
\end{aligned}
$$

where $B(\cdot, \cdot)$ denotes the Archimedean Beta function.

By using $B\left(z_{1}+\epsilon, z_{2}+\delta\right) \leq B\left(z_{1}, z_{2}\right)$ for $\epsilon, \delta \geq 0$,

$$
\begin{aligned}
B\left(1, \frac{\alpha+(m-1)(\alpha-n) v-\alpha_{j}}{\alpha-n}\right) & \leq B(v, m v), \\
B\left(\frac{\alpha-\alpha_{k}}{\alpha-n}, \frac{\alpha-n+(m-1)(\alpha-n) v}{\alpha-n}\right) & \leq B(v, m v),
\end{aligned}
$$

and

$$
(t-\tau)^{\frac{\left.(m-1)(\alpha-n) v-\alpha_{r}+\alpha\right)}{\alpha-n}}=(t-\tau)^{m v-\frac{(\alpha-n) v+\alpha_{r}-\alpha}{\alpha-n}} \leq C(t-\tau)^{m v}
$$

for $1 \leq r \leq N+1$, we get

$$
\begin{aligned}
\left|R_{m+1}(x, t, \xi, \tau)\right| \leq & C(2 N+2)^{m} \frac{(\Gamma(v))^{m}}{\Gamma((m+1) v)}(t-\tau)^{m v} \\
& \times \sum_{k=1}^{N+1}\left[(t-\tau)^{1 /(\alpha-n)}+\|x-\xi\|_{p}\right]^{-\alpha_{k}}
\end{aligned}
$$




\section{$\S 6$. Uniqueness of the solution}

We recall that $\widetilde{\mathcal{E}}$ is the $\mathbb{C}$-vector space of all functions $\varphi: \mathbb{Q}_{p}^{n} \rightarrow \mathbb{C}$ such that there exists a ball $B_{l}^{n}$, with $l$ depending only on $\varphi$, such that $\varphi\left(x+x^{\prime}\right)=\varphi(x)$ for any $x \in \mathbb{Q}_{p}^{n}$ and $x^{\prime} \in B_{l}^{n}$. Notice that $\mathfrak{M}_{\lambda} \subset \widetilde{\mathcal{E}}$ for any $\lambda$. We identify each element of $\widetilde{\mathcal{E}}$ with a distribution on $\mathbb{Q}_{p}^{n}$. We now recall the following fact: $T \in S^{\prime}$ with $\operatorname{supp}(T) \subset B_{N}^{n}$ if and only if $\widehat{T} \in \widetilde{\mathcal{E}}$ and its parameter of local constancy is greater than $-N$ (cf. [17, p. 109]).

Lemma 6.1. $\mathbf{W}_{\alpha}: \widetilde{\mathcal{E}} \rightarrow \widetilde{\mathcal{E}}$ is a well-defined linear operator. Furthermore,

$$
\left(\mathbf{W}_{\alpha} \varphi\right)(x)=-\mathcal{F}_{\xi \rightarrow x}^{-1}\left(A_{w_{\alpha}}\left(\|\xi\|_{p}\right) \mathcal{F}_{x \rightarrow \xi} \varphi\right) .
$$

Proof. Let $l$ be a parameter of local constancy of $\varphi$. Then

$$
\begin{aligned}
\left(\mathbf{W}_{\alpha} \varphi\right)(x) & =\int_{\|y\|_{p} \geq p^{l}} \frac{\varphi(x-y)-\varphi(x)}{w_{\alpha}\left(\|y\|_{p}\right)} d^{n} y \\
& =\frac{1_{\mathbb{Q}_{p}^{n} \backslash B_{l}^{n}}(x)}{w_{\alpha}\left(\|x\|_{p}\right)} * \varphi(x)-\varphi(x) \int_{\|y\|_{p} \geq p^{l}} \frac{d^{n} y}{w_{\alpha}\left(\|y\|_{p}\right)} .
\end{aligned}
$$

Then by taking the Fourier transform in $S^{\prime}$ we get

$$
\mathcal{F}\left(\mathbf{W}_{\alpha} \varphi\right)(\xi)=\left(\int_{\mathbb{Q}_{p}^{n}} 1_{\mathbb{Q}_{p}^{n} \backslash B_{l}^{n}}(x) \frac{\Psi(x \cdot \xi)-1}{w_{\alpha}\left(\|x\|_{p}\right)} d^{n} x\right)(\mathcal{F} \varphi)(\xi),
$$

and since $\mathcal{F} \varphi \in S^{\prime}$ with $\operatorname{supp}(\mathcal{F} \varphi) \subset B_{-l}^{n}$,

$$
\mathcal{F}\left(\mathbf{W}_{\alpha} \varphi\right)(\xi)=\left(\int_{\mathbb{Q}_{p}^{n}} \frac{\Psi(x \cdot \xi)-1}{w_{\alpha}\left(\|x\|_{p}\right)} d^{n} x\right)(\mathcal{F} \varphi)(\xi) .
$$

Therefore,

$$
\left(\mathbf{W}_{\alpha} \varphi\right)(x)=-\mathcal{F}_{\xi \rightarrow x}^{-1}\left(A_{w_{\alpha}}\left(\|\xi\|_{p}\right) \mathcal{F}_{x \rightarrow \xi} \varphi\right) \in \widetilde{\mathcal{E}} .
$$

Take $\gamma$ to be a real number such that $\lambda<\gamma<\alpha_{1}-n<\cdots<\alpha_{N}-n<\alpha-n$, fix an integer $L$, and set $\psi(x):=p^{L n} \Omega\left(p^{L}\|x\|_{p}\right) *\|x\|_{p}^{\gamma}$. Then

$$
\psi(x)= \begin{cases}\|x\|_{p}^{\gamma} & \text { if }\|x\|_{p}>p^{-L} \\ C & \text { if }\|x\|_{p} \leq p^{-L}\end{cases}
$$

and thus $\psi \in \widetilde{\mathcal{E}}$.

Lemma 6.2. With the above notation, there exist positive constants $C_{1}$ and $C_{2}$ such that

(i) $\left|\left(\mathbf{W}_{\alpha} \psi\right)(x)\right| \leq C_{1}\|x\|_{p}^{\alpha-\gamma+n}$,

(ii) $\left|\left(\mathbf{W}_{\alpha_{k}} \psi\right)(x)\right| \leq C_{2}\|x\|_{p}^{\alpha_{k}-\gamma+n}$ for $k=1, \ldots, N$. 
Proof. By Lemma 6.1,

$$
\left(\mathbf{W}_{\alpha} \psi\right)(x)=-\mathcal{F}_{\xi \rightarrow x}^{-1}\left(A_{w_{\alpha}}\left(\|\xi\|_{p}\right) \Omega\left(p^{-L}\|\xi\|_{p}\right) \frac{\|\xi\|_{p}^{-\gamma-n}}{\Gamma_{n}(n+\gamma)}\right) \quad \text { in } S^{\prime}
$$

where $\Gamma_{n}(n+\gamma)=\frac{1-p^{\gamma}}{1-p^{-\gamma-n}}$. Now, since $A_{w_{\alpha}}\left(\|\xi\|_{p}\right) \Omega\left(p^{-L}\|\xi\|_{p}\right)\|\xi\|_{p}^{-\gamma-n} / \Gamma_{n}(n+\gamma)$ is radial and locally integrable, by applying the formula for the Fourier transform of a radial function (see e.g. [17, Example 8, p. 43]) we get

$$
\begin{aligned}
& \left(\mathbf{W}_{\alpha} \varphi\right)(x) \\
& =\frac{-\|x\|_{p}^{-n}}{\Gamma_{n}(n+\gamma)}\left[\left(1-p^{-n}\right)\|x\|_{p}^{\gamma+n} \sum_{j=0}^{\infty} A_{w_{\alpha}}\left(\|x\|_{p}^{-1} p^{-j}\right) \Omega\left(\|x\|_{p}^{-1-j} p^{-j}\right) p^{j(\gamma+n)-j n}\right. \\
& \left.\quad-A_{w_{\alpha}}\left(\|x\|_{p} p^{-j}\right) \Omega\left(\|x\|_{p}^{-1} p^{-L+1}\right)\|x\|_{p}^{\gamma+n}\right]
\end{aligned}
$$

as a distribution on $\mathbb{Q}_{p}^{n} \backslash\{0\}$. Now by using [7, Lemma 3.4],

$$
\left|\left(\mathbf{W}_{\alpha} \varphi\right)(x)\right| \leq C^{\prime}\left[\left(1-p^{-n}\right) \sum_{j=0}^{\infty} p^{-j(\alpha-n)+j \gamma}-p^{(-L+1)(\alpha-n)}\right]\|x\|_{p}^{-\alpha+n+\gamma} .
$$

The proof of (ii) is similar.

Theorem 6.3. Assume that the coefficients $a_{k}(x, t), k=0,1, \ldots, N$ are nonnegative bounded continuous functions, $b(x, t)$ is a bounded continuous function, $0 \leq \lambda<\alpha_{1}-n, \alpha>n+1$ (if $a_{1}(x, t)=\cdots=a_{k}(x, t) \equiv 0$, we suppose that $0 \leq \lambda<\alpha-n)$ and $u(x, t)$ is a solution of the Cauchy problem (5.1) with $f(x, t)=$ $\varphi(x) \equiv 0$ that belongs to the class $\mathfrak{M}_{\lambda}$. Then $u(x, t) \equiv 0$.

Proof. We may assume that $b(x, t) \geq 0$, otherwise we take $u(x, t) e^{\lambda t}$ with $\lambda>$ $b(x, t)$. We first prove that $u(x, t) \geq 0$. For contradiction, suppose that $u\left(x^{\prime}, t^{\prime}\right)<0$ for some $x^{\prime} \in \mathbb{Q}_{p}^{n}$ and $t^{\prime} \in(0, T]$. By Lemma 6.2 , it follows that $\left(\mathbf{W}_{\alpha} \psi\right)(x) \rightarrow 0$ and $\left(\mathbf{W}_{\alpha_{k}} \psi\right)(x) \rightarrow 0$ as $\|x\|_{p} \rightarrow \infty$, and thus

$$
M:=\sup _{\substack{0 \leq t \leq T \\ x \in \mathbb{Q}_{p}^{n}}}\left\{a_{0}(x, t)\left|\left(\mathbf{W}_{\alpha} \psi\right)(x)\right|+\sum_{k=1}^{N} a_{k}(x, t)\left|\left(\mathbf{W}_{\alpha_{k}} \psi\right)(x)\right|\right\}<\infty .
$$

We pick $\rho>0$ such that $u\left(x^{\prime}, t^{\prime}\right)+T \rho<0$, and then $\sigma>0$ such that

$$
\begin{aligned}
& u\left(x^{\prime}, t^{\prime}\right)+T \rho+\sigma \psi\left(x^{\prime}\right)<0, \\
& \rho-\sigma M<0 .
\end{aligned}
$$

We now consider the function $v(x, t):=u(x, t)+t \rho+\sigma \psi(x)$. From (6.2), it follows that $v\left(x^{\prime}, t^{\prime}\right)<0$, so that

$$
\inf _{0 \leq t \leq T, x \in \mathbb{Q}_{p}^{n}} v(x, t)<0
$$


On the other hand, since $u(\cdot, t) \in \mathfrak{M}_{\lambda}$, we have $\lim _{\|x\|_{p} \rightarrow \infty} u(x, t) / \psi(x)=0$ and thus $\lim _{\|x\|_{p} \rightarrow \infty} v(x, t)>0$ for any $t>0$. This implies that there exist $x_{0} \in \mathbb{Q}_{p}^{n}$ and $t_{0} \in(0, T]$ such that

$$
\inf _{0 \leq t \leq T, x \in \mathbb{Q}_{p}^{n}} v(x, t)=\min _{0 \leq t \leq T, x \in \mathbb{Q}_{p}^{n}} v(x, t)=v\left(x_{0}, t_{0}\right)<0,
$$

and thus, by formula (3.1), $\left(\mathbf{W}_{\alpha} v\right)\left(x_{0}, t_{0}\right) \geq 0,\left(\mathbf{W}_{\alpha_{k}} v\right)\left(x_{0}, t_{0}\right) \geq 0$ for all $k$, and $\frac{\partial v}{\partial t}\left(x_{0}, t_{0}\right) \leq 0$, hence

$$
\begin{aligned}
\frac{\partial v}{\partial t}\left(x_{0}, t_{0}\right)-a_{0}(x, t)\left(\mathbf{W}_{\alpha} v\right)\left(x_{0}, t_{0}\right)-\sum_{k=1}^{N} a_{k}(x, t)\left(\mathbf{W}_{\alpha_{k}} v\right)\left(x_{0}, t_{0}\right) & \\
+b(x, t) v\left(x_{0}, t_{0}\right) & <0 .
\end{aligned}
$$

Now, by (6.3),

$$
\begin{array}{r}
\frac{\partial v}{\partial t}(x, t)-a_{0}(x, t)\left(\mathbf{W}_{\alpha} v\right)(x, t)-\sum_{k=1}^{N} a_{k}(x, t)\left(\mathbf{W}_{\alpha_{k}} v\right)(x, t)+b(x, t) v(x, t) \\
=\rho-\sigma\left[a_{0}(x, t)\left(\mathbf{W}_{\alpha} \psi\right)(x)+\sum_{k=1}^{N} a_{k}(x, t)\left(\mathbf{W}_{\alpha_{k}} \psi\right)(x)\right]+b(x, t)[\rho t+\sigma \psi(x)] \\
\geq \rho-\sigma M>0 .
\end{array}
$$

We have obtained a contradiction, thus $u(x, t) \geq 0$. Finally, taking $-u(x, t)$ instead of $u(x, t)$, we conclude that $u(x, t) \equiv 0$.

\section{$\S 7$. Markov processes}

In this section we show that the fundamental solution $\Lambda(x, t, \xi, \tau)$ of the Cauchy problem (5.1) is the transition density of a Markov process. We need some preliminary results.

Lemma 7.1. If the coefficients $a_{k}(x, t)$ and $b(x, t)$ are nonnegative, then $\Lambda(x, t, \xi, \tau) \geq 0$.

Proof. It is sufficient to show that $u(x, t)=\int_{\mathbb{Q}_{p}^{n}} \Lambda(x, t, \xi, \tau) \varphi(\xi) d^{n} \xi \geq 0$, where $u(x, t)$ is the solution of (5.1) with $f(x, t) \equiv 0$, and initial condition $u(x, 0)=$ $\varphi(x) \geq 0$ with $\varphi \in S\left(\mathbb{Q}_{p}^{n}\right)$. From (5.12), (5.13), and Lemma 4.3(iii), it follows that

$$
u(x, t) \rightarrow 0 \quad \text { as }\|x\|_{p} \rightarrow \infty .
$$

Now, if $u(x, t)<0$, then there exist $x_{0} \in \mathbb{Q}_{p}^{n}$ and $t_{0} \in(0, T]$ such that

$$
\inf _{0 \leq t \leq T, x \in \mathbb{Q}_{p}^{n}} u(x, t)=u\left(x_{0}, t_{0}\right)<0 .
$$


This implies that $\left(\mathbf{W}_{\alpha} u\right)\left(x_{0}, t_{0}\right) \geq 0,\left(\mathbf{W}_{\alpha_{k}} u\right)\left(x_{0}, t_{0}\right) \geq 0$ for all $k$, and $\frac{\partial u}{\partial t}\left(x_{0}, t_{0}\right)$ $\leq 0$. On the other hand,

$$
\frac{\partial u}{\partial t}(x, t)-a_{0}(x, t)\left(\mathbf{W}_{\alpha} u\right)(x, t)-\sum_{k=1}^{N} a_{k}(x, t)\left(\mathbf{W}_{\alpha_{k}} u\right)(x, t)=0 .
$$

By using the uniform parabolicity condition $a_{0}(x, t) \geq \mu>0$, we get $\left(\mathbf{W}_{\alpha} u\right)\left(x_{0}, t_{0}\right)$ $=0$; then by $(3.1), u\left(x, t_{0}\right)$ is constant, and by $(7.1), u\left(x, t_{0}\right) \equiv 0$, which contra$\operatorname{dicts}(7.2)$.

Lemma 7.2. If $b(x, t) \equiv 0$, then $\int_{\mathbb{Q}_{p}^{n}} \Lambda(x, t, \xi, \tau) d^{n} \xi=1$.

Proof. By integrating (5.14) in the variable $\xi$ over the whole space $\mathbb{Q}_{p}^{n}$, and by using Lemma 4.9 (iii), we have $\frac{\partial}{\partial t}\left(\int_{\mathbb{Q}_{p}^{n}} \Lambda(x, t, \xi, \tau) d^{n} \xi\right)=0$, thus $\int_{\mathbb{Q}_{p}^{n}} \Lambda(x, t, \xi, \tau) d^{n} \xi$ is independent of $t$. Now, by integrating (5.12) over the whole space $\mathbb{Q}_{p}^{n}$ in the variable $\xi$ and by using Lemma 4.3 (iv), we have

$$
\int_{\mathbb{Q}_{p}^{n}} \Lambda(x, t, \xi, \tau) d^{n} \xi=1+\int_{\tau}^{t} \int_{\mathbb{Q}_{p}^{n}} \int_{\mathbb{Q}_{p}^{n}} Z(x-\eta, t-\theta ; \eta, \theta) \phi(\eta, \theta, \xi, \tau) d^{n} \eta d^{n} \xi d \theta .
$$

The result is obtained by taking $t=\tau$ in the above formula.

Lemma 7.3. If $b(x, t) \equiv 0$ and $f(x, t) \equiv 0$, then

$$
\Lambda(x, t, \xi, \tau)=\int_{\mathbb{Q}_{p}^{n}} \Lambda(x, t, y, \sigma) \Lambda(y, \sigma, \xi, \tau) d^{n} y
$$

Proof. Consider the following initial value problem:

$$
\left\{\begin{array}{l}
\frac{\partial u}{\partial t}(x, t)-a_{0}(x, t)\left(\mathbf{W}_{\alpha} u\right)(x, t)-(\widetilde{\mathbf{W}} u)(x, t)=0, \\
u(x, \tau)=\varphi(x), \quad x \in \mathbb{Q}_{p}^{n} \text { and } t \in(\tau, \sigma] .
\end{array}\right.
$$

By Theorem 5.5, $u(x, \sigma)=\int_{\mathbb{Q}_{p}^{n}} \Lambda(x, \sigma, \xi, \tau) \varphi(\xi) d^{n} \xi$. Now consider

$$
\left\{\begin{array}{l}
\frac{\partial u}{\partial t}(x, t)-a_{0}(x, t)\left(\mathbf{W}_{\alpha} u\right)(x, t)-(\widetilde{\mathbf{W}} u)(x, t)=0, \\
u(x, \sigma)=\int_{\mathbb{Q}_{p}^{n}} \Lambda(x, \sigma, \xi, \tau) \varphi(\xi) d^{n} \xi, \quad x \in \mathbb{Q}_{p}^{n}, t \in(\sigma, T], \tau<\sigma<T .
\end{array}\right.
$$

By Theorem 5.5 and Fubini's Theorem, the solution of (7.5) is given by

$$
u(x, t)=\int_{\mathbb{Q}_{p}^{n}}\left(\int_{\mathbb{Q}_{p}^{n}} \Lambda(x, t, y, \sigma) \Lambda(y, \sigma, \xi, \tau) d^{n} y\right) \varphi(\xi) d^{n} \xi .
$$

On the other hand, (7.5) is equivalent to

$$
\left\{\begin{array}{l}
\frac{\partial u}{\partial t}(x, t)-a_{0}(x, t)\left(\mathbf{W}_{\alpha} u\right)(x, t)-(\widetilde{\mathbf{W}} u)(x, t)=0 \\
u(x, \tau)=\varphi(x), \quad x \in \mathbb{Q}_{p}^{n}, t \in(\tau, T]
\end{array}\right.
$$


which has a solution given by $u(x, t)=\int_{\mathbb{Q}_{p}^{n}} \Lambda(x, t, \xi, \tau) \varphi(\xi) d^{n} \xi$. Now, by Theorem 6.3 ,

$$
\int_{\mathbb{Q}_{p}^{n}} \Lambda(x, t, \xi, \tau) \varphi(\xi) d^{n} \xi=\int_{\mathbb{Q}_{p}^{n}}\left(\int_{\mathbb{Q}_{p}^{n}} \Lambda(x, t, y, \sigma) \Lambda(y, \sigma, \xi, \tau) d^{n} y\right) \varphi(\xi) d^{n} \xi
$$

for any test function $\varphi$, which implies (7.3).

Theorem 7.4. If the coefficients $a_{k}(x, t), k=1, \ldots, N$, are non-negative bounded continuous functions, $b(x, t) \equiv 0,0 \leq \lambda<\alpha_{1}-n, \alpha>n+1$ (if $a_{1}(x, t)=\cdots=$ $a_{k}(x, t) \equiv 0$, we suppose that $\left.0 \leq \lambda<\alpha-n\right)$, and $f(x, t) \equiv 0$, then the fundamental solution $\Lambda(x, t, \xi, \tau)$ is the transition density of a bounded right-continuous Markov process without second kind discontinuities.

Proof. The result follows from [10, Theorem 3.6] by using Lemmas 7.1-7.3 and $5.12-5.13$, and Lemma 4.3(iii).

\section{$\S 8$. The Cauchy problem is well-posed}

In this section, we study the continuity of the solution of the Cauchy problem (5.1) with respect to $\varphi(x)$ and $f(x, t)$. We assume that the coefficients $a_{k}(x, t), k=$ $0,1, \ldots, N$, are non-negative bounded continuous functions, $b(x, t)$ is a bounded continuous function, $0 \leq \lambda<\alpha_{1}-n$ (if $a_{1}(x, t)=\cdots=a_{k}(x, t) \equiv 0$, we suppose that $0 \leq \lambda<\alpha-n), \varphi \in \mathfrak{M}_{\lambda}$ and $f(\cdot, t) \in \mathfrak{M}_{\lambda}$ uniformly in $t$, with $0 \leq \lambda<\alpha_{1}-n$.

We identify $\mathfrak{M}_{\lambda}$ with the $\mathbb{R}$-vector space of all functions " $\phi(\cdot, t) \in \mathfrak{M}_{\lambda}$ uniformly in $t$," and introduce on $\mathfrak{M}_{\lambda}$ the following norm:

$$
\|\phi\|_{\mathfrak{M}_{\lambda}}:=\sup _{t \in[0, T]} \sup _{x \in \mathbb{Q}_{p}^{n}}\left|\frac{\phi(x, t)}{1+\|x\|_{p}^{\lambda}}\right| .
$$

From now on, we consider $\mathfrak{M}_{\lambda}$ as a topological vector space with the topology induced by $\|\cdot\|_{\mathfrak{M}_{\lambda}}$. We also consider $\mathfrak{M}_{\lambda} \times \mathfrak{M}_{\lambda}$ as a topological vector space with the topology induced by the norm $\|\cdot\|_{\mathfrak{M}_{\lambda}}+\|\star\|_{\mathfrak{M}_{\lambda}}$.

Theorem 8.1. With the above hypotheses, consider the following operator:

$$
\mathfrak{M}_{\lambda} \times \mathfrak{M}_{\lambda} \stackrel{L}{\rightarrow} \mathfrak{M}_{\lambda}, \quad(\varphi(\cdot), f(\cdot, t)) \mapsto u(\cdot, t),
$$

where $u(x, t)$ is given by (5.11). Then $\|u(\cdot, t)\|_{\mathfrak{M}_{\lambda}} \leq C\left(\|\varphi(\cdot)\|_{\mathfrak{M}_{\lambda}}+\|f(\cdot, t)\|_{\mathfrak{M}_{\lambda}}\right)$, i.e. $\boldsymbol{L}$ is a continuous operator.

Proof. We write $u(x, t)=u_{1}(x, t)+u_{2}(x, t)$ where

$u_{1}(x, t)=\int_{0}^{t} \int_{\mathbb{Q}_{p}^{n}} \Lambda(x, t, \xi, \tau) f(\xi, \tau) d^{n} \xi d \tau \quad$ and $\quad u_{2}(x, t)=\int_{\mathbb{Q}_{p}^{n}} \Lambda(x, t, \xi, 0) \varphi(\xi) d^{n} \xi$ 
as before. Now

$$
\begin{aligned}
& \left|u_{1}(x, t)\right| \leq \int_{0}^{t} \int_{\mathbb{Q}_{p}^{n}}|\Lambda(x, t, \xi, \tau)||f(\xi, \tau)| d^{n} \xi d \tau \\
& \quad \leq\|f(x, t)\|_{\mathfrak{M}_{\lambda}}\left\{\int_{0}^{t} \int_{\mathbb{Q}_{p}^{n}}|\Lambda(x, t, \xi, \tau)| d^{n} \xi d \tau+\int_{0}^{t} \int_{\mathbb{Q}_{p}^{n}}|\Lambda(x, t, \xi, \tau)|\|\xi\|_{p}^{\lambda} d^{n} \xi d \tau\right\}
\end{aligned}
$$

and by (5.12)-(5.13), (5.3) and Proposition 4.4,

$$
\begin{array}{r}
\left|u_{1}(x, t)\right| \leq C_{0}\|f(\cdot, t)\|_{\mathfrak{M}_{\lambda}}\left\{\int_{0}^{t}(t-\tau)^{1+\frac{n-\alpha}{\alpha-n}} d \tau+\int_{0}^{t}(t-\tau)^{2-\frac{\lambda}{\alpha-n}+\frac{n-\alpha}{\alpha-n}} d \tau\right. \\
+\sum_{k=1}^{N+1} \int_{0}^{t}(t-\tau)^{1+\frac{n-\alpha_{k}}{\alpha-n}} d \tau+\left(1+\|x\|_{p}^{\lambda}\right) \int_{0}^{t}(t-\tau)^{1+\frac{n-\alpha}{\alpha-n}} d \tau \\
\left.+\left(1+\|x\|_{p}^{\lambda}\right) \int_{0}^{t}(t-\tau)^{2-\frac{\lambda}{\alpha-n}+\frac{n-\alpha}{\alpha-n}} d \tau+\left(1+\|x\|_{p}^{\lambda}\right) \sum_{k=1}^{N+1} \int_{0}^{t}(t-\tau)^{1+\frac{n-\alpha_{k}}{\alpha-n}} d \tau\right\} \\
\leq\|f(\cdot, t)\|_{\mathfrak{M}_{\lambda}}\left\{C_{1}(T)+C_{2}(T)\left(1+\|x\|_{p}^{\lambda}\right)\right\} .
\end{array}
$$

Hence,

Similarly, one shows that

$$
\left|\frac{u_{1}(x, t)}{1+\|x\|_{p}^{\lambda}}\right| \leq\|f(\cdot, t)\|_{\mathfrak{M}_{\lambda}}\left\{\frac{C_{1}(T)}{1+\|x\|_{p}^{\lambda}}+C_{2}(T)\right\} .
$$

$$
\left|\frac{u_{2}(x, t)}{1+\|x\|_{p}^{\lambda}}\right| \leq\|\varphi(\cdot)\|_{\mathfrak{M}_{\lambda}}\left\{\frac{C_{1}^{\prime}(T)}{1+\|x\|_{p}^{\lambda}}+C_{2}^{\prime}(T)\right\},
$$

therefore $\|u(\cdot, t)\|_{\mathfrak{M}_{\lambda}} \leq C\left(\|\varphi(\cdot)\|_{\mathfrak{M}_{\lambda}}+\|f(\cdot, t)\|_{\mathfrak{M}_{\lambda}}\right)$.

\section{Acknowledgements}

The authors wish to thank the anonymous referee for his/her careful reading of the original manuscript, and they also wish to thank Sergii Torba for many useful comments and discussions during the preparation of this article.

\section{References}

[1] S. Albeverio and W. Karwowski, Jump processes on leaves of multibranching trees, J. Math. Phys. 49 (2008), no. 9, 093503, 20 pp. Zbl 1152.81310 MR 2455842

[2] S. Albeverio, A. Yu. Khrennikov and V. M. Shelkovich, Theory of p-adic distributions: linear and nonlinear models, Cambridge Univ. Press, 2010. Zbl 1198.46001 MR 2641698

[3] V. A. Avetisov, A. H. Bikulov, S. V. Kozyrev and V. A. Osipov, p-adic models of ultrametric diffusion constrained by hierarchical energy landscapes, J. Phys. A 35 (2002), 177-189. Zbl 1038.82077 MR 1945917 
[4] V. A. Avetisov, A. Kh. Bikulov and V. A. Osipov, p-adic description of characteristic relaxation in complex systems, J. Phys. A 36 (2003), 4239-4246. Zbl 1049.82051 MR 1984499

[5] R. F. Bass and D. A. Levin, Transition probabilities for symmetric jump processes, Trans. Amer. Math. Soc. 354 (2002), 2933-2953. Zbl 0993.60070 MR 1895210

[6] O. F. Casas-Sánchez and W. A. Zúñiga-Galindo, p-adic elliptic quadratic forms, parabolictype pseudodifferential equations with variable coefficients and Markov processes, $p$-Adic Numbers Ultrametric Anal. Appl. 6 (2014), 1-20. Zbl 06416125 MR 3162769

[7] L. F. Chacón-Cortes and W. A. Zúñiga-Galindo, Nonlocal operators, parabolic-type equations, and ultrametric random walks, J. Math. Phys. 54 (2013), 113503. Zbl 1288.82027 MR 3137041

[8] Z.-Q. Chen and T. Kumagai, Heat kernel estimates for jump processes of mixed types on metric measure spaces, Probab. Theory Related Fields 140 (2008), 277-317. Zbl 1131.60076 MR 2357678

[9] B. Dragovich, A. Yu. Khrennikov, S. V. Kozyrev and I. V. Volovich, On p-adic mathematical physics, p-Adic Numbers Ultrametric Anal. Appl. 1 (2009), 1-17. Zbl 1187.81004 MR 2566116

[10] E. B. Dynkin, Markov processes. Vol. I, Springer, 1965. Zbl 0132.37901 MR 0193671

[11] A. Friedman, Partial differential equations of parabolic type, Prentice-Hall, Englewood Cliffs, NJ, 1964. Zbl 0144.34903 MR 0181836

[12] W. Karwowski, Diffusion processes with ultrametric jumps, Rep. Math. Phys. 60 (2007), 221-235. Zbl 1210.60092 MR 2374819

[13] J. Kigami, Transitions on a noncompact Cantor set and random walks on its defining tree, Ann. Inst. H. Poincaré Probab. Statist. 49 (2013), 1090-1129. Zbl 1286.31006 MR 3127915

[14] A. N. Kochubei, Pseudo-differential equations and stochastics over non-Archimedean fields, Dekker, New York, 2001. Zbl 0984.11063 MR 1848777

[15] J. J. Rodríguez-Vega, On a general type of $p$-adic parabolic equations, Rev. Colombiana Mat. 43 (2009), 101-114. Zbl 1206.35269 MR 2602049

[16] J. J. Rodríguez-Vega and W. A. Zúñiga-Galindo, Taibleson operators, $p$-adic parabolic equations and ultrametric diffusion, Pacific J. Math. 237 (2008), 327-347. Zbl 1232.35201 MR 2421125

[17] V. S. Vladimirov, I. V. Volovich and E. I. Zelenov, p-adic analysis and mathematical physics, World Sci., 1994. Zbl 0864.46048 MR 1349578

[18] W. A. Zúñiga-Galindo, Parabolic equations and Markov processes over $p$-adic fields, Potential Anal. 28 (2008), 185-200. Zbl 1134.35005 MR 2373104 